\title{
The mimetic finite difference method for the Landau-Lifshitz equation
}

\author{
Eugenia Kim ${ }^{\mathrm{b}, \mathrm{a}}$, Konstantin Lipnikov ${ }^{\mathrm{a}}$ \\ ${ }^{a}$ MS-B284, Los Alamos National Laboratory, Los Alamos, NM 87544 \\ ${ }^{b}$ Department of Mathematics, University of California, Berkeley, CA 94720
}

\begin{abstract}
The Landau-Lifshitz equation describes the dynamics of the magnetization inside ferromagnetic materials. This equation is highly nonlinear and has a non-convex constraint (the magnitude of the magnetization is constant) which pose interesting challenges in developing numerical methods. We develop and analyze explicit and implicit mimetic finite difference schemes for this equation. These schemes work on general polytopal meshes which provide enormous flexibility to model magnetic devices with various shapes. A projection on the unit sphere is used to preserve the magnitude of the magnetization. We also provide a proof that shows the exchange energy is decreasing in certain conditions. The developed schemes are tested on general meshes that include distorted and randomized meshes. The numerical experiments include a test proposed by the National Institute of Standard and Technology and a test showing formation of domain wall structures in a thin film.
\end{abstract}

Keywords: micromagnetics, Landau-Lifshitz equation, Landau-Lifshitz-Gilbert equation, mimetic finite difference method, polygonal meshes

\section{Introduction}

Micromagnetics studies behavior of ferromagnetic materials at sub-micrometer length scales [20]. These scales are large enough to use a continuum PDE model and are small enough to resolve important magnetic structures such as domain walls, vortices and skyrmions [35]. The dynamics of the magnetic distribution $\boldsymbol{m}$ in a ferromagnetic material is governed by the Landau-Lifshitz (LL) equation. There exist several equivalent forms of the LL equation, such as the Landau-Lifshitz-Gilbert equation, that lead to a large family of numerical nethods.

The evolution of $\boldsymbol{m}$ is driven by the effective field $\mathbf{h}$ which can be described as the functional derivative of the LL energy density with respect to the magnetization. The LL energy is given by

$$
E(\mathbf{m})=\frac{\eta}{2} \int_{V}|\nabla \mathbf{m}|^{2} \mathrm{~d} x+\frac{Q}{2} \int_{V}\left(m_{2}^{2}+m_{3}^{2}\right) \mathrm{d} x-\frac{1}{2} \int_{V} \mathbf{h}_{\mathbf{s}} \cdot \mathbf{m} \mathrm{d} x-\int_{V} \mathbf{h}_{\mathbf{e}} \cdot \mathbf{m} \mathrm{d} x .
$$

The first term is the exchange energy, which favors the alignment of the magnetization along a common direction. The second term is the anisotropy energy, which prefers the certain orientation of the spins due to the crystalline lattice. The third term is the stray field energy, 
which is induced by the magnetization distribution inside the material. The last term is the external field energy which favors the orientation of the spins along an external field. The exchange energy, anisotropy energy and the external field energy are local terms in that a local change in the magnetization affects only locally, whereas the stray field energy is the nonlocal term in that a local change in the magnetization affects globally [15].

The LL equation has a few important properties [15]. First, the magnitude of the magnetization is preserved, namely $|\mathbf{m}|=M$. We can renormalize the LL equation so that $M=1$. Secondly, the energy decreases in time, in case of a constant applied field, which is called the Lyapunov structure. Lastly, if there is no damping, i.e. $\alpha=0$, the energy is conserved, which is called the Hamiltonian structure.

Various numerical methods have been developed for the LL equation, see e.g. review papers [15, 23, 33. The discretization strategies in space are discussed in the following articles : In [41], the finite difference methods based on the field and energy are presented. The field-based finite difference method is obtained by discretizing field $\mathbf{h}$ itself, whereas in the energy based approach this field is derived from the discretized energy. A finite element method is employed in [20]. The magnetization is approximated with piecewise linear functions and the effective field $\mathbf{h}$ is obtained as the first variation of the discretized energy. In [44, the finite element method with piecewise linear functions is applied to the Landau-Lifshitz-Gilbert equation, which is another formulation of the LL equation.

A large family of time stepping schemes have been developed which conserve the magnitude of the magnetization. The Gauss-Seidel projection method developed in [46, 47, 24] uses another formulation of the LL equation (the last equation in $(2.8)$ ) and treats $|\nabla \boldsymbol{m}|^{2}$ as the Lagrange multiplier for the pointwise constraint $|\boldsymbol{m}|=1$. The gyromagnetic and damping terms are treated separately to overcome the difficulties associated with the stiffness and nonlinearity. The resulting method is first-order accurate and unconditionally stable. In [30], the semi-analytic integration method is developed by analytically integrating the system of ODEs appearing after a spatial discretization of the LL equation. This method is the first-order accurate but explicit, hence is subject to a Courant time step constraint. The geometric integration method has been applied in [31], and in a more general setting in [34], using the Cayley transform to lift the LL equation to the Lie algebra of the three dimensional rotation group. Unlike the semi-analytic integration methods, this method is more amenable for building numerical schemes with higher-order accuracy. Finally, we mention the method based on the mid-point rule [10, 17] which is second-order accurate, unconditionally stable, and preserves the magnitude of the magnetization, as well as the Lyapunov and Hamiltonian structures of the LL equation. Although these methods could be extended to finite element discretizations, to the best of our knowledge, the literature has only examples of finite difference schemes, which are difficult to use for general domains.

The semi-discrete schemes are introduced in [43] for 2D and in [14 for 3D formulation of the LL equation and error estimates are derived under the assumption that there exist a strong solution.

The finite element methods for the LL equation are typically presented with rigorous convergence analysis that deals with weak solutions. In [5, 4, 7], the finite element method is developed for an equivalent formulation of the LL equation (see, formula (2.7) ) which is the first-order accurate (in the energy norm) in both space and time and requires only one linear solver on each time step. In [32, 6], the method is developed further to achieve 
the second-order accuracy in time. In [8], Bartels and Prohl considered an implicit time integration method for the Landau-Lifshitz-Gilbert equation (see, formula (2.6)), which is unconditionally stable, but a nonlinear solver is needed on each time step. In [16], Cimrák proposed a scheme for the LL equation, using a midpoint rule that could be easily adapted to the limiting cases, but a nonlinear solver is needed on each time step.

We present explicit and implicit mimetic finite difference (MFD) schemes [9] for the LL equation. In contrast to the existing numerical methods that use conventional spatial discretizations with various time stepping strategies, we deliver a new spatial discretization which has a number of unique properties. First, it works on arbitrary polytopal (polygonal in 2D and polyhedral in 3D) meshes including locally refined meshes with degenerate cells. For the same mesh resolution, polytopal meshes need fewer cells to cover the domain than simplicial meshes, which leads to fewer number of unknowns and a more efficient scheme. Elegant treatment of degenerate cells that appear in adaptive mesh refinement/coarsening algorithms allows us to track accurate dynamics of domain walls. Although mesh adaptation is beyond the focus of this paper, we illustrate the underlying idea with one numerical experiment. Secondly, we use a mixed formulation of the LL equation that simplifies numerical control of the constraint $|\mathbf{m}|=1$. To the best of our knowledge, the schemes proposed in this paper are the first ones that are based on a mixed formulation of the LL equation. Thirdly, the MFD could be applied to problems posed in general domains, like the finite element methods, which is a key advantage compared to the finite difference methods. To the best of our knowledge, the time stepping schemes such as GSPM [46, 47, 24] and other geometric methods [30, 31, 34, 10, 17] have only been tested on finite difference stencils. Fourthly, we prove that the exchange energy decreases on polygonal meshes under certain conditions, which were not addressed in GSPM [46, 47, 24] and other methods [30, 31, 34, 10]. Fifthly, our implicit scheme has the similar complexity as the algorithms in Alouges [5, 4, 7, in that we need to solve a linear system for each time step. Finally, compared to the methods in [5, 4, 7, 8], this method is developed for the LL equation, which makes it more suitable to apply to the limiting cases, which is important for physical simulations.

The MFD method was originally designed to preserve or mimic important mathematical and physical properties of continuum PDEs in discrete schemes on unstructured polytopal meshes. It has been successfully employed for solving diffusion, convection-diffusion, electromagnetic, and linear elasticity problems and for modeling various fluid flows. The original MFD method is a low-order method, but miscellaneous approaches were developed towards higher-order methods. We refer to book [9] and review paper [37] for extensive review of mimetic schemes. This the first application of the mimetic discretization technology to a geometric dispersive partial differential equation.

The mimetic discretization framework combines rich tools of a finite element analysis with the flexibility of finite volume meshes. Here, we consider explicit and implicit time integration schemes and demonstrate the flexibility of this framework with various numerical experiments. We perform stability analysis on polygonal meshes but defer rigorous convergence analysis for future work.

The computation of the stray field term is the most time consuming part of each micromagnetic simulation due to its nonlocal nature. Numerous numerical methods for the stray field calculation are described and studied in [2]. They can be divided into two groups. The first group includes methods that solve a PDE in $\mathbb{R}^{d}$ for the potential field. Since this PDE is 
posed in an infinite domain, hybrid numerical methods are typically used. The finite element and boundary element methods are used in [21, 26], the finite element method and the shell transformation are used in [13. The computation cost is reduced by using multigrid preconditioners 45 and $\mathcal{H}$-matrix approximation [42]. The second group includes methods based on direct evaluation of the integral with a nonlocal kernel, e.g. the fast Fourier transform [40, 3, 49, 25], the fast multipole method [11], nonuniform grid method [39], and the tensor grid method [18]. The best numerical methods reach complexity between $N$ and $N \log N$, where $N$ is the number of unknowns.

The paper is organized as follows. In Section 2, we describe the PDE formulation of the LL equation. In Section 3, we present the MFD method. In Subection 3.5, the computation of the stray field is reviewed based on [48]. In Section 4, the stability of the explicit and implicit schemes is analyzed. Finally, in Section 5, we verify and validate the proposed schemes using analytical solutions, the NIST $\mu$ mag standard problem 4 , and the test showing formation of domain wall structures in a thin film.

\section{Problem formulation}

The dynamics of the magnetic distribution in a ferromagnetic material occupying a region $\Omega \subset \mathbb{R}^{d}$ where $d=2$, or 3 , is governed by the LL equation, see, e.g. (5.4). After its normalization, we obtain the following PDE for the magnetization $\mathbf{m}: \Omega \times[0, T] \rightarrow \mathbb{R}^{3}$ :

$$
\frac{\partial \mathbf{m}}{\partial t}=-\mathbf{m} \times \mathbf{h}-\alpha \mathbf{m} \times(\mathbf{m} \times \mathbf{h}),
$$

where $\alpha$ is the dimensionless damping parameter and $\mathbf{h}$ is the effective field. The first term on the right hand side is the gyromagnetic term and the second term is the damping term. The problem is closed by imposing the Neumann or Dirichlet boundary conditions and initial conditions.

It is immediately to see that $|\mathbf{m}|$ is constant in time, so we assume that $|\mathbf{m}|=1$. The effective field is defined as the functional derivative of the LL energy density:

$$
\mathbf{h}(\mathbf{m})=-\frac{\delta E(\mathbf{m})}{\delta \mathbf{m}}=\eta \triangle \mathbf{m}-Q\left(m_{2} e_{2}+m_{3} e_{3}\right)+\mathbf{h}_{\mathbf{s}}(\mathbf{m})+\mathbf{h}_{\mathbf{e}}
$$

where $\eta$ is the exchange constant, $Q$ is an anisotropy constant, $\mathbf{h}_{s}$ is the stray field, and $\mathbf{h}_{e}$ is an external field. Let us collect the low-order terms (with respect to $\Delta \mathbf{m}$ ) in a single variable

$$
\overline{\mathbf{h}}(\mathbf{m})=-Q\left(m_{2} e_{2}+m_{3} e_{3}\right)+\mathbf{h}_{\mathbf{s}}(\mathbf{m})+\mathbf{h}_{\mathbf{e}} .
$$

These terms are usually regarded as the low-order terms when considering mathematical properties such as the existence and regularity of the solution [5]. Since constant $\eta$ is not critical for the description of the mimetic scheme, we set $\eta=1$.

The stray field is given by $\mathbf{h}_{\mathbf{s}}=-\nabla \phi$. Let $\Omega^{c}$ denote the complement of $\Omega$. Then, the potential $\phi$ satisfies (see [23] for more detail):

$$
\begin{aligned}
\triangle \phi & = \begin{cases}\nabla \cdot \mathbf{m} & \text { in } \Omega, \\
0 & \text { on } \Omega^{c},\end{cases} \\
{[\phi]_{\partial \Omega} } & =0, \\
{[\nabla \phi \cdot \boldsymbol{n}]_{\partial \Omega} } & =-\mathbf{m} \cdot \mathbf{n},
\end{aligned}
$$


where $[v]_{\partial \Omega}$ denotes a jump of function $v$ across the domain boundary, and $\boldsymbol{n}$ is the unit normal vector. Hence, the stray field $\mathbf{h}_{\mathbf{s}}$ is given by

$$
\mathbf{h}_{\mathbf{s}}(x)=-\frac{1}{4 \pi} \nabla \int_{\Omega} \nabla\left(\frac{1}{|x-y|}\right) \cdot \mathbf{m}(y) \mathrm{d} y .
$$

Note, that there are several equivalent forms of the LL equation, e.g. the Landau-LifshitzGilbert equation:

$$
\frac{\partial \mathbf{m}}{\partial t}-\alpha \mathbf{m} \times \frac{\partial \mathbf{m}}{\partial t}=-\left(1+\alpha^{2}\right)(\mathbf{m} \times \mathbf{h}) .
$$

Another equivalent form, used in [5] to develop a numerical scheme, is

$$
\alpha \frac{\partial \mathbf{m}}{\partial t}+\mathbf{m} \times \frac{\partial \mathbf{m}}{\partial t}=\left(1+\alpha^{2}\right)(\mathbf{h}-(\mathbf{h} \cdot \mathbf{m}) \mathbf{m}) .
$$

In a special case of $\overline{\mathbf{h}}=0$, we have $\mathbf{h}=\Delta \mathbf{m}$. Then, the simplified LL equation 2.1 has more equivalent forms:

$$
\begin{aligned}
\frac{\partial \mathbf{m}}{\partial t} & =-\mathbf{m} \times \Delta \mathbf{m}-\alpha \mathbf{m} \times(\mathbf{m} \times \Delta \mathbf{m}) \\
& =-\mathbf{m} \times \Delta \mathbf{m}+\alpha \Delta \mathbf{m}-\alpha(\mathbf{m} \cdot \Delta \mathbf{m}) \mathbf{m} \\
& =-\mathbf{m} \times \Delta \mathbf{m}+\alpha \Delta \mathbf{m}+\alpha|\nabla \mathbf{m}|^{2} \mathbf{m} .
\end{aligned}
$$

Here, we used the vector identity $\mathbf{a} \times(\mathbf{b} \times \mathbf{c})=(\mathbf{a} \cdot \mathbf{c}) \mathbf{b}-(\mathbf{a} \cdot \mathbf{b}) \mathbf{c},|\mathbf{m}|=1$, and

$$
\mathbf{m} \cdot \frac{\partial \mathbf{m}}{\partial u}=0, \quad u \in\{x, y, z\} \text {. }
$$

If we consider only the damping term on the right-hand side of 2.8 we obtain $\frac{\partial \mathbf{m}}{\partial t}=$ $-\alpha \mathbf{m} \times(\mathbf{m} \times \triangle \mathbf{m})$, which is called the harmonic map heat flow into $\mathbb{S}^{2}$ [27]. If we consider only the gyromagnetic term on the right-hand side of 2.8 , we obtain $\frac{\partial \mathbf{m}}{\partial t}=-\mathbf{m} \times \triangle \mathbf{m}$, which is called the Schrödinger map, a geometric generalization of the linear Schrödinger equation [27]. By using the LL equation to design a numerical scheme, instead of the Landau-LifshitzGilbert equation (2.6) or (2.7), we can immediately apply it to the harmonic map heat flow and the Schrödinger map.

\section{Mimetic discretization of the Landau-Lifshitz equation}

In this section, we apply the mimetic finite difference (MFD) method to the LL equation. Let us introduce the magnetic flux tensor $\mathbf{p}=-\nabla \mathbf{m}$. Then,

$$
\frac{\partial \mathbf{m}}{\partial t}=\mathbf{m} \times \operatorname{div} \mathbf{p}+\alpha \mathbf{m} \times(\mathbf{m} \times \operatorname{div} \mathbf{p})+\mathbf{f}(\mathbf{m}) .
$$

where

$$
\mathbf{f}(\mathbf{m})=-\mathbf{m} \times \overline{\mathbf{h}}(\mathbf{m})-\alpha \mathbf{m} \times(\mathbf{m} \times \overline{\mathbf{h}}(\mathbf{m}))
$$

corresponds to the low-order terms (2.3). The MFD method solves simultaneously for both $\mathbf{m}$ and $\mathbf{p}$. It mimics duality of the divergence and gradient operators in the discrete setting. 
This property is used in the stability analysis. The MFD method works on arbitrary polygonal or polyhedral mesh which provides enormous flexibility for modeling non-rectangular mechanical devices.

Let the computational domain $\Omega$ be decomposed into $N_{E}$ non-overlapping polygonal or polyhedral elements $E$ with the maximum diameter $h$. Let $N_{F}$ denote the total number of mesh edges (faces in 3D). We use $|E|$ to denote the area (volume in 3D) of $E$. Similarly, $|f|$ denotes the length of mesh edge $f$ (area of mesh face $f$ in $3 \mathrm{D}$ ). Let $\boldsymbol{n}_{E}$ be the unit vector normal to $\partial E$.

To define degrees of freedom for the mimetic scheme, we assume that $\mathbf{m} \in \mathcal{Q}$ and $\mathbf{p} \in \mathcal{F}$, where $\mathcal{Q}=\left(L^{2}(\Omega)\right)^{3}$ and

$$
\mathcal{F}=\left\{\mathbf{p} \mid \mathbf{p} \in\left(L^{s}(\Omega)\right)^{d \times 3}, s>2, \operatorname{div} \mathbf{p} \in\left(L^{2}(\Omega)\right)^{3}\right\} .
$$

\subsection{Global mimetic formulation}

Let $\mathbf{m}=\left(m_{x}, m_{y}, m_{z}\right)$. The degrees of freedom for each component of the magnetization are associated with elements $E$ and denoted as $m_{x, E}, m_{y, E}$, and $m_{z, E}$. They represent the mean values of $\mathbf{m}$ :

$$
m_{u, E}=\frac{1}{|E|} \int_{E} m_{u} \mathrm{~d} x, \quad u \in\{x, y, z\} .
$$

Thus, control of the magnetization magnitude reduces to simple cell-based constraints $\left|\mathbf{m}_{E}\right|=$ 1. Consider the vector space

$$
\mathcal{Q}^{h}=\left\{m_{u}^{h}: m_{u}^{h}=\left(m_{u, E_{1}}, \cdots, m_{u, E_{N_{E}}}\right)^{T}\right\} .
$$

The dimension of this space is equal to the number of mesh elements. Then, the discrete magnetization $\mathbf{m}^{h}=\left(m_{x}^{h}, m_{y}^{h}, m_{z}^{h}\right)$ with $m_{u}^{h} \in \mathcal{Q}^{h}$.

Let $\mathbf{p}=\left(\mathbf{p}_{x}, \mathbf{p}_{y}, \mathbf{p}_{z}\right)$. The degrees of freedom for each component of the magnetic flux are associated with mesh edges $f$ (faces in $3 \mathrm{D}$ ) and denoted as $p_{x, E, f}, p_{y, E, f}$ and $p_{z, E, f}$. They represent the mean normal flux across edge $f$ of element $E$ :

$$
p_{u, E, f}=\frac{1}{|f|} \int_{f} \mathbf{p}_{u} \cdot \boldsymbol{n}_{E} \mathrm{~d} x, \quad u \in\{x, y, z\} .
$$

We need a few notations for local groups of degrees of freedom. Let $p_{u, E}$ be the vector of degrees of freedom associated with element $E$ and $\mathbf{p}_{E}=\left(p_{x, E}, p_{y, E}, p_{z, E}\right)^{T}$. Consider the vector space

$$
\mathcal{F}^{h}=\left\{p_{u}^{h}: p_{u}^{h}=\left(p_{u, E_{1}}, \cdots, p_{u, E_{N_{E}}}\right)^{T}\right\}
$$

The dimension of this space is equal to twice the number of internal mesh edges (faces in 3D) plus the number of boundary edges. Then, the discrete magnetic flux $\mathbf{p}^{h}=\left(p_{x}^{h}, p_{y}^{h}, p_{z}^{h}\right)$ with $p_{u}^{h} \in \mathcal{F}^{h}$.

In the global mimetic formulation we consider the reduced space for discrete fluxes still denoted by $\mathcal{F}^{h}$. Let $f$ be an internal edge shared by two elements $E_{1}$ and $E_{2}$. The reduced space satisfies the flux continuity constraints

$$
p_{u, E_{1}, f}+p_{u, E_{2}, f}=0
$$


for all internal edges. The reduced space allows us to work with exterior normal vectors $\mathbf{n}_{E}$ which simplifies some formulas. In a computer code, the flux continuity constraints are used to reduce the problem size.

The degrees of freedom were chosen to define the discrete divergence operator easily. For each component $u$ of the magnetic flux, the divergence theorem for element $E$ leads to the discrete divergence operator:

$$
\mathcal{D} \mathcal{I} \mathcal{V}_{E} p_{u, E}=\frac{1}{|E|} \sum_{f \in \partial E}|f| p_{u, E, f}
$$

Let

$$
\operatorname{DIV}_{E} \mathbf{p}_{E}=\left(\mathcal{D} \mathcal{I} \mathcal{V}_{E} p_{x, E}, \mathcal{D} \mathcal{I} \mathcal{V}_{E} p_{y, E}, \mathcal{D} \mathcal{I} \mathcal{V}_{E} p_{z, E}\right)^{T}
$$

The MFD method builds the discrete gradient operator from the discrete duality principle. Recall that in the continuum setting, under the homogeneous boundary conditions, we have the Green formula:

$$
\int_{\Omega} \mathbf{m} \cdot \operatorname{div} \mathbf{p} \mathrm{d} x=-\int_{\Omega} \nabla \mathbf{m}: \mathbf{p} \mathrm{d} x
$$

It states that the gradient operator is negatively adjoint to the divergence operator with respect to the $L^{2}$-inner products. In the MFD method, we mimic this formula. First, we replace the $L^{2}$-inner products by discrete inner products in spaces of the degrees of freedom. In the space of discrete magnetizations, we define the following inner product:

$$
\left[\mathbf{m}^{h}, \mathbf{w}^{h}\right]_{\mathcal{Q}}=\sum_{u \in\{x, y, z\}}\left[m_{u}^{h}, w_{u}^{h}\right]_{\mathcal{Q}}, \quad\left[m_{u}^{h}, w_{u}^{h}\right]_{\mathcal{Q}}=\sum_{E \in \Omega_{h}}\left[m_{u, E}^{h}, w_{u, E}^{h}\right]_{\mathcal{Q}, E} .
$$

Since we have only one degree of freedom per mesh element, we have $\left[m_{u, E}^{h}, w_{u, E}^{h}\right]_{\mathcal{Q}, E}=$ $|E| m_{u, E} w_{u, E}$. In the space of discrete fluxes, we define the following inner product:

$$
\left[\mathbf{p}^{h}, \mathbf{q}^{h}\right]_{\mathcal{F}}=\sum_{u \in\{x, y, z\}}\left[p_{u}^{h}, q_{u}^{h}\right]_{\mathcal{F}}, \quad\left[p_{u}^{h}, q_{u}^{h}\right]_{\mathcal{F}}=\sum_{E \in \Omega_{h}}\left[p_{u, E}, q_{u, E}\right]_{\mathcal{F}, E}
$$

where $[\cdot, \cdot]_{\mathcal{F}, E}$ is an element-based inner product that requires special construction discussed later. The mimetic gradient operator is defined implicitly from the discrete duality property:

$$
\left[\mathbf{m}^{h}, \text { DIV } \mathbf{p}^{h}\right]_{\mathcal{Q}}=-\left[\text { GRAD } \mathbf{m}^{h}, \mathbf{p}^{h}\right]_{\mathcal{F}} \quad \forall \mathbf{m}^{h}, \mathbf{p}^{h}
$$

Due to basic properties of the inner products, the mimetic gradient is defined uniquely.

The semi-discrete mimetic formulation is to find $\mathbf{m}^{h}$ and $\mathbf{p}^{h}$ such that

$$
\begin{aligned}
\mathbf{p}^{h} & =-\mathbf{G R A D} \mathbf{m}^{h} \\
\frac{\partial \mathbf{m}^{h}}{\partial t}+\alpha \mathbf{D I V} \mathbf{p}^{h} & =\mathbf{m}^{h} \times \mathbf{D I V} \mathbf{p}^{h}+\alpha\left(\mathbf{m}^{h} \cdot \mathbf{D I V} \mathbf{p}^{h}\right) \mathbf{m}^{h}+\mathbf{f}^{h}\left(\mathbf{m}^{h}\right)
\end{aligned}
$$

where $\mathbf{f}^{h}\left(\mathbf{m}^{h}\right)$ is a discretization of equation (3.1). Non-homogeneous boundary conditions can be incorporated in the MFD framework using the approach described in [29]. In the next section, we describe another way to include boundary conditions. 


\subsection{Local mimetic formulation}

For the local mimetic formulation, the starting point is the local Green formula:

$$
\int_{E} \mathbf{m} \cdot \operatorname{div} \mathbf{p} \mathrm{d} x=-\int_{E} \nabla \mathbf{m}: \mathbf{p} \mathrm{d} x+\int_{\partial E}(\mathbf{p} \cdot \mathbf{n}) \cdot \mathbf{m} \mathrm{d} x
$$

To discretize the last integral, we use additional degrees of freedom for magnetization on mesh edges $f$ that we denote by $\mathbf{m}_{f}=\left(m_{x, f}, m_{y, f}, m_{z, f}\right)$. Let $\widetilde{\mathbf{m}}_{E}$ be the vector of the additional degrees of freedom associated with element $E$. The total number of these degrees of freedom is equal to the number of mesh edges, $N_{F}$, times three. The local mimetic gradient operator is defined implicitly from the discrete duality property:

$$
\left[\mathbf{m}_{E}, \operatorname{DIV}_{E} \mathbf{p}_{E}\right]_{\mathcal{Q}, E}=-\left[\operatorname{GRAD}_{E}\left(\begin{array}{c}
\mathbf{m}_{E} \\
\widetilde{\mathbf{m}}_{E}
\end{array}\right), \mathbf{p}_{E}\right]_{\mathcal{F}, E}+\sum_{f \in \partial E}|f| \mathbf{p}_{f} \cdot \mathbf{m}_{f}
$$

for all $\mathbf{m}_{E}, \widetilde{\mathbf{m}}_{E}$, and $\mathbf{p}_{E}$. Due to basic properties of the inner products, the local mimetic gradient operator is defined uniquely. Moreover, by our assumption, all inner products are sums of inner products for vector components. This allows us to define a component-wise gradient operator via

$$
\left[m_{u, E}, \mathcal{D} \mathcal{I} \mathcal{V}_{E} p_{u, E}\right]_{\mathcal{Q}, E}=-\left[\mathcal{G} \mathcal{R} \mathcal{A} \mathcal{D}_{E}\left(\begin{array}{c}
m_{u, E} \\
\widetilde{m}_{u, E}
\end{array}\right), p_{u, E}\right]_{\mathcal{F}, E}+\sum_{f \in \partial E}|f| p_{u, E, f} \cdot m_{u, f}
$$

The last formula can be simplified if we define inner product matrices $\mathbb{M}_{\mathcal{Q}, E}$ and $\mathbb{M}_{\mathcal{F}, E}$ :

$$
\left[m_{u, E}, w_{u, E}\right]_{\mathcal{Q}, E}=m_{u, E} \mathbb{M}_{\mathcal{Q}, E} w_{u, E}, \quad\left[p_{u, E}, q_{u, E}\right]_{\mathcal{F}, E}=p_{u, E} \mathbb{M}_{\mathcal{F}, E} q_{u, E}
$$

The first matrix is simply the number $|E|$. The construction of the second matrix is more involved and is discussed in subsection 3.4. If the boundary of element $E$ has $n$ faces $f_{i}$, we obtain the following explicit formula for the mimetic gradient:

$$
\mathcal{G} \mathcal{R} \mathcal{A D}_{E}\left(\begin{array}{c}
m_{u, E} \\
\widetilde{m}_{u, E}
\end{array}\right)=\mathbb{M}_{\mathcal{F}, E}^{-1}\left(\begin{array}{c}
\left|f_{1}\right|\left(m_{u, f_{1}}-m_{u, E}\right) \\
\left|f_{2}\right|\left(m_{u, f_{2}}-m_{u, E}\right) \\
\vdots \\
\left|f_{n}\right|\left(m_{u, f_{n}}-m_{u, E}\right)
\end{array}\right)
$$

The semi-discrete mimetic formulation is to find $\mathbf{m}_{E}, \widetilde{\mathbf{m}}_{E}$, and $\mathbf{p}_{E}$, for $E \in \Omega^{h}$, such that

$$
\begin{aligned}
\mathbf{p}_{E} & =-\mathbf{G R A D}_{E}\left(\begin{array}{c}
\mathbf{m}_{E} \\
\widetilde{\mathbf{m}}_{E}
\end{array}\right), \\
\frac{\partial \mathbf{m}_{E}}{\partial t}+\alpha \mathbf{D I V}_{E} \mathbf{p}_{E} & =\mathbf{m}_{E} \times \mathbf{D I V} \mathbf{I V}_{E}+\alpha\left(\mathbf{m}_{E} \cdot \mathbf{D I V}_{E} \mathbf{p}_{E}\right) \mathbf{m}_{E}+\mathbf{f}_{E}\left(\mathbf{m}_{E}\right),
\end{aligned}
$$

subject to continuity (3.5), boundary, and initial conditions. The Dirichlet boundary conditions are imposed by prescribing given values to the auxiliary magnetization unknowns on mesh edges. The Neumann boundary conditions are imposed by setting magnetic fluxes to given values.

The local mimetic formulation implies the global one. Equivalence of the local and global gradient operators can be shown by summing up equations 3.10 and observing that interface term are canceled out due to flux continuity conditions (3.5). The local formulation is more convenient for a computer implementation. The global formulation is more convenient for convergence analysis. 


\subsection{Implicit-explicit time discretization}

To discretize time derivative in (3.12), we consider the $\theta$-scheme. Let $k$ denote the time step and superscript $j$ denote the time moment $t^{j}=j k$. Then, the $\theta$-scheme is

$$
\begin{gathered}
\mathbf{p}_{E}^{j+\theta}=-\mathbf{G R A D}_{E}\left(\begin{array}{c}
\mathbf{m}_{E}^{j+\theta} \\
\widetilde{\mathbf{m}}_{E}^{j+\theta}
\end{array}\right) \\
\frac{\mathbf{m}_{E}^{j+1}-\mathbf{m}_{E}^{j}}{k}+\alpha \mathbf{D I V} \mathbf{V}_{E} p_{E}^{j+\theta}=\mathbf{m}_{E}^{j} \times \mathbf{D I V}_{E} \mathbf{p}_{E}^{j+\theta}+\alpha\left(\mathbf{m}_{E}^{j} \cdot\left(\mathbf{D I V}_{E} \mathbf{p}_{E}^{j+\theta}\right)\right) \mathbf{m}_{E}^{j}+\mathbf{f}_{E}^{j}\left(\mathbf{m}_{E}^{j}\right) .
\end{gathered}
$$

Note that $\theta=0$ for the explicit scheme and $\theta=1$ for the implicit scheme. The later scheme is not fully implicit and a single linear solver is required to advance the solution. Hereafter, we consider only $\theta=1$. Hereafter, we drop superscript ' $h$ ' and write $\mathbf{m}^{j}$ instead of $\mathbf{m}^{h, j}$. Inserting the first equation in the second one and in the flux continuity conditions (multiplied by $|f|$ for better symmetry), and imposing boundary and initial conditions, we obtain a system of algebraic equations for cell-based and edge-based magnetizations:

$$
\mathbb{A}^{j}\left(\begin{array}{c}
\mathbf{m}^{j+1} \\
\widetilde{\mathbf{m}}^{j+1}
\end{array}\right)=\left(\begin{array}{l}
\mathbf{b}_{1}^{j} \\
\mathbf{b}_{2}^{j}
\end{array}\right)
$$

The right-hand side vector depends on $\mathbf{m}^{j}$, external field, stray field, and boundary conditions. The stiffness matrix $\mathbb{A}^{j}$ is sparse and could be written as the sum of local matrices,

$$
\mathbb{A}^{j}=\sum_{E \in \Omega^{h}} \mathbb{N}_{E} \mathbb{A}_{E}^{j} \mathbb{N}_{E}^{T} .
$$

where $\mathbb{N}_{E}$ is the conventional assembly matrix which maps local indices to global indices. We present detailed structure of the local matrix which is important for analyzing its structural properties and selecting optimal solver. Let us consider an element $E$ with $n$ edges located strictly inside the computational domain. The corresponding local matrix has size $3(n+1) \times$ $3(n+1)$ and the following block structure:

$$
\mathbb{A}_{E}^{j}=\left(\begin{array}{ll}
\mathbb{A}^{E E} & \mathbb{A}^{E f} \\
\mathbb{A}^{f E} & \mathbb{A}^{f f}
\end{array}\right)
$$

where the first block row corresponds to three cell-based magnetizations. Let $\mathbb{I}$ denote a generic identity matrix, $\mathbb{C}_{E}$ be the $n \times n$ diagonal matrix, $\mathbb{C}_{E}=\operatorname{diag}\left\{\left|f_{1}\right|, \ldots,\left|f_{n}\right|\right\}$, and $\mathbf{e}=(1,1, \ldots, 1)^{T}$. Using the tensor-product notation to represent matrices, we have

$$
\begin{aligned}
\mathbb{A}^{E E} & =\mathbb{I}+\frac{k}{|E|}\left(\mathbf{e}^{T} \mathbb{C}_{E} \mathbb{M}_{\mathcal{F}, E}^{-1} \mathbb{C}_{E} \mathbf{e}\right) \hat{\mathbb{A}}^{j}, & & \mathbb{A}^{E f}=-\frac{k}{|E|}\left(\mathbf{e}^{T} \mathbb{C}_{E} \mathbb{M}_{\mathcal{F}, E}^{-1} \mathbb{C}_{E}\right) \otimes \hat{\mathbb{A}}^{j} \\
\mathbb{A}^{f E} & =-\left(\mathbb{C}_{E} \mathbb{M}_{\mathcal{F}, E}^{-1} \mathbb{C}_{E} \mathbf{e}\right) \otimes \mathbb{I}, & \mathbb{A}^{f f} & =\left(\mathbb{C}_{E} \mathbb{M}_{\mathcal{F}, E}^{-1} \mathbb{C}_{E}\right) \otimes \mathbb{I},
\end{aligned}
$$

and

$$
\hat{\mathbb{A}}^{j}=\alpha \mathbb{I}-\alpha \mathbf{m}_{E}^{j}\left(\mathbf{m}_{E}^{j}\right)^{T}-\left(\begin{array}{ccc}
0 & -m_{z, E}^{j} & m_{y, E}^{j} \\
m_{z, E}^{j} & 0 & -m_{x, E}^{j} \\
-m_{y, E}^{j} & m_{x, E}^{j} & 0
\end{array}\right)
$$


To preserve the geometric constraint $\left|\mathbf{m}_{E}^{j+1}\right|=1$, we need to modify the solution by projecting it onto the unit sphere. This leads to the following algorithm.

Algorithm 1. For a given final time $T>0$, set $J=\left[\frac{T}{k}\right]$.

1. Set an initial discrete magnetization $\mathbf{m}^{0}$ at the centers of mesh elements.

2. For $j=0, \ldots, J-1$,

(a) Form and solve the system $(3.14)$. Let $\left(\begin{array}{c}\hat{\mathbf{m}}^{j+1} \\ \widetilde{\mathbf{m}}^{j+1}\end{array}\right)$ denote the solution.

(b) Renormalize the element-centered magnetizations:

$$
\mathbf{m}_{E}^{j+1}:=\frac{\hat{\mathbf{m}}_{E}^{j+1}}{\left|\hat{\mathbf{m}}_{E}^{j+1}\right|}, \quad \forall E \in \Omega^{h}
$$

Finally, note that the scheme require only matrix $\mathbb{M}_{\mathcal{F}, E}^{-1}$. In the next section, we show that it could be calculated directly for the same cost as matrix $\mathbb{M}_{\mathcal{F}, E}$.

\subsection{Construction of matrices $\mathbb{M}_{\mathcal{F}, E}$ and $\mathbb{M}_{\mathcal{F}, E}^{-1}$}

The construction of the matrices $\mathbb{M}_{\mathcal{F}, E}$ and $\mathbb{M}_{\mathcal{F}, E}^{-1}$ is based on the consistency and stability conditions discussed in detail in [12]. Here, we briefly summarize the underlying ideas. The inner product matrix $\mathbb{M}_{\mathcal{F}, E}$ must represent an accurate quadrature for the continuum $L^{2}$ inner product of two functions. More specifically, we require that the following consistency condition (also known as the exactness property) holds:

$$
\left(p_{u, E}^{0}\right)^{T} \mathbb{M}_{\mathcal{F}, E} q_{u, E}=\left[p_{u, E}^{0}, q_{u, E}\right]_{\mathcal{F}, E}=\int_{E} \mathbf{p}_{u}^{0} \cdot \mathbf{q}_{u} \mathrm{~d} x
$$

for any constant vector function $\mathbf{p}_{u}^{0}$ and any sufficiently smooth function $\mathbf{q}_{u}$ with constant divergence and constant normal components on element edges. Here $p_{u, E}^{0}$ and $q_{u, E}$ are vectors of the degrees of freedom for functions $\mathbf{p}_{u}^{0}$ and $\mathbf{q}_{u}$, respectively. Writing $\mathbf{p}_{u}^{0}$ as the gradient of a linear function $m_{u}^{1}$ with mean zero value on $E$ and integrating by parts, we obtain:

$$
\int_{E} \mathbf{p}_{u}^{0} \cdot \mathbf{q}_{u} \mathrm{~d} x=-\int_{E} m_{u}^{1} \operatorname{div} \mathbf{q}_{u} \mathrm{~d} x+\int_{\partial E}\left(\mathbf{q}_{u} \cdot \mathbf{n}_{E}\right) m_{u}^{1} \mathrm{~d} x=\sum_{f \in \partial E} q_{u, E, f} \int_{f} m_{u}^{1} \mathrm{~d} x .
$$

For a given $\mathbf{p}_{u}^{0}$, this formula uses the degrees of freedom and the computable edge integrals. Taking three linearly independent constant vector functions ( such as $\mathbf{p}_{u}^{0}=(1,0,0)^{T}$ ), inserting them in the exactness property, and using the definition of the degrees of freedom, we obtain the matrix equation

$$
\mathbb{M}_{\mathcal{F}, E} \mathbb{N}=\mathbb{R}
$$

where

$$
\mathbb{N}=\left(\begin{array}{ccc}
n_{x_{f_{1}}} & n_{y_{f_{1}}} & n_{z_{f_{1}}} \\
n_{x_{f_{2}}} & n_{y_{f_{2}}} & n_{z_{f_{2}}} \\
\vdots & \vdots & \vdots \\
n_{x_{f_{m}}} & n_{y_{f_{m}}} & n_{z_{f_{m}}}
\end{array}\right), \quad \mathbb{R}=\left(\begin{array}{ccc}
\left|f_{1}\right|\left(x_{f_{1}}-x_{E}\right) & \left|f_{1}\right|\left(y_{f_{1}}-y_{E}\right) & \left|f_{1}\right|\left(z_{f_{1}}-z_{E}\right) \\
\left|f_{2}\right|\left(x_{f_{2}}-x_{E}\right) & \left|f_{2}\right|\left(y_{f_{2}}-y_{E}\right) & \left|f_{2}\right|\left(z_{f_{2}}-z_{E}\right) \\
\vdots & \vdots & \vdots \\
\left|f_{m}\right|\left(x_{f_{m}}-x_{E}\right) & \left|f_{m}\right|\left(y_{f_{m}}-y_{E}\right) & \left|f_{m}\right|\left(z_{f_{m}}-z_{E}\right)
\end{array}\right) .
$$


Here $n_{u_{f}}$ is the $u$-th component of the outward unit normal vector to edge $f$ of $E,\left(x_{f}, y_{f}, z_{f}\right)$ is the edge centroid, and $\left(x_{E}, y_{E}, z_{E}\right)$ is the element centroid. It was shown in [12] that the symmetric positive definite matrix

$$
\mathbb{M}_{\mathcal{F}, E}=\frac{1}{|E|} \mathbb{R}^{T}+\gamma\left(\mathbb{I}-\mathbb{N}\left(\mathbb{N}^{T} \mathbb{N}\right)^{-1} \mathbb{N}^{T}\right), \quad \gamma>0
$$

is a solution to matrix equation (3.19). The matrix $\mathbb{M}_{\mathcal{F}, E}$ looks like a mass matrix when both terms in (3.20) are scaled equally with respect to local mesh size. Since the second term includes the orthogonal projection, we can simply set $\gamma=|E|$ or $\gamma=\frac{1}{2|E|} \operatorname{trace}\left(\mathbb{R} \mathbb{R}^{T}\right)$.

The general solution to $(3.19)$ is

$$
\mathbb{M}_{\mathcal{F}, E}=\frac{1}{|E|} \mathbb{R}^{T}+\left(\mathbb{I}-\mathbb{N}\left(\mathbb{N}^{T} \mathbb{N}\right)^{-1} \mathbb{N}^{T}\right) \mathbb{G}\left(\mathbb{I}-\mathbb{N}\left(\mathbb{N}^{T} \mathbb{N}\right)^{-1} \mathbb{N}^{T}\right)
$$

where $\mathbb{G}$ is a symmetric positive definite matrix. Mathematically, the selection of this matrix must satisfy the stability condition: There exists two positive constants $c_{0}, C_{0}>0$, independent of $h$ and $E$, such that, for every $q_{u} \in \mathcal{F}_{E}^{h}$ and every $E \in \Omega^{h}$, we have

$$
c_{0}|E| q_{u, E}^{T} q_{u, E} \leq q_{u, E}^{T} \mathbb{M}_{\mathcal{F}, E} q_{u, E}=\left[q_{u, E}, q_{u, E}\right]_{\mathcal{F}, E} \leq C_{0}|E| q_{u, E}^{T} q_{u, E} .
$$

Note that the matrix equation $(3.19)$ can be also written as

$$
\mathbb{M}_{\mathcal{F}, E}^{-1} \mathbb{R}=\mathbb{N} .
$$

Since the role of matrices $\mathbb{R}$ and $\mathbb{N}$ has changed but the equation structure did not, we can write immediately the general solution to this equation:

$$
\mathbb{M}_{\mathcal{F}, E}^{-1}=\frac{1}{|E|} \mathbb{N N}^{T}+\left(\mathbb{I}-\mathbb{R}\left(\mathbb{R}^{T} \mathbb{R}\right)^{-1} \mathbb{R}^{T}\right) \tilde{\mathbb{G}}\left(\mathbb{I}-\mathbb{R}\left(\mathbb{R}^{T} \mathbb{R}\right)^{-1} \mathbb{R}^{T}\right)
$$

where $\tilde{\mathbb{G}}$ is symmetric positive definite matrix. In a computer code, we can use a scalar matrix $\tilde{\mathbb{G}}=\tilde{\gamma} \mathbb{I}$ where $\tilde{\gamma}=\frac{1}{|E|}$ or $\tilde{\gamma}=\frac{1}{2|E|} \operatorname{trace}\left(\mathbb{N} \mathbb{N}^{T}\right)$.

Remark 1. Since both matrix equations (3.19) and (3.23) have multiple solutions, formulas (3.20) and (3.24) with $\gamma=\tilde{\gamma}^{-1}=|E|$ represent non-related members from two families of solutions.

\subsection{Computation of the stray field}

In this subsection, we discuss how to compute the stray field $\mathbf{h}_{s}(\mathbf{m})$ on various meshes and specifically on uniform meshes over rectangular domains. For general geometries, we refer to the stray field calculation survey [2].

We can break the integral in equation 2.5 into sum over mesh elements,

$$
\begin{aligned}
\mathbf{h}_{s}(x) & =-\frac{1}{4 \pi} \sum_{E \in \Omega^{h}} \nabla \int_{E} \nabla\left(\frac{1}{|x-y|}\right) \cdot \mathbf{m}(y) \mathrm{d} y \\
& =\frac{1}{4 \pi} \sum_{E \in \Omega^{h}} \nabla\left\{\int_{E} \frac{\nabla \cdot \mathbf{m}(y)}{|x-y|} \mathrm{d} y-\int_{\partial E} \frac{\mathbf{m}(y) \cdot \mathbf{n}_{E}}{|x-y|} \mathrm{d} y\right\} .
\end{aligned}
$$


The discrete magnetization is constant in each element $E$; hence, its divergence is zero and we obtain the following formula for calculating the discrete stray field:

$$
\mathbf{h}_{s, E}=\frac{1}{4 \pi} \sum_{E \in \Omega^{h}} \int_{\partial E} \frac{x_{E}-y}{\left|x_{E}-y\right|^{3}} \mathbf{m}_{E}(y) \cdot \mathbf{n}_{E} \mathrm{~d} y .
$$

Efficient implementation of the above summation can be done on the uniform mesh over a rectangular domain. We applied this method for a few micromagnetic simulations considered in subsection 5.3 and 5.4 . Let $E_{i j k}$ be a cuboid element,

$$
E_{i j k}=\left[x_{i-\frac{1}{2}}, x_{i+\frac{1}{2}}\right] \times\left[y_{j-\frac{1}{2}}, y_{j+\frac{1}{2}}\right] \times\left[z_{k-\frac{1}{2}}, z_{k+\frac{1}{2}}\right]
$$

where $x_{i}=(i-0.5) h_{x}, y_{j}=(j-0.5) h_{y}$, and $z_{k}=(k-0.5) h_{z}$. Then, the above summation can be rewritten using a $3 \times 3$ demagnetization tensor $\mathbb{K}$ :

$$
\mathbf{h}_{s, E_{i j k}}=\sum_{p, q, r} \mathbb{K}_{i-p, j-q, k-r} \mathbf{m}_{E_{p q r}}
$$

The demagnetization tensor can be computed analytically:

$$
\mathbb{K}_{i-p, j-q, k-r}=-\frac{1}{4 \pi} \sum_{s_{x}, s_{y}, s_{z}} s_{x} s_{y} s_{z}\left(\begin{array}{ccc}
\arctan \left(\frac{R_{y} R_{z}}{R_{x} R}\right) & -\log \left(R+R_{z}\right) & -\log \left(R+R_{y}\right) \\
-\log \left(R+R_{z}\right) & \arctan \left(\frac{R_{x} R_{z}}{R_{y} R}\right) & -\log \left(R+R_{x}\right) \\
-\log \left(R+R_{y}\right) & -\log \left(R+R_{x}\right) & \arctan \left(\frac{R_{x} R_{y}}{R_{z} R}\right)
\end{array}\right)
$$

where $s_{x}, s_{y}, s_{z}= \pm 1, R_{x}=\frac{h_{x}}{2}-s_{x}(i-p) h_{x}, R_{y}=\frac{h_{y}}{2}-s_{y}(j-q) h_{y}, R_{z}=\frac{h_{z}}{2}-s_{z}(k-r) h_{z}$ and $R=\sqrt{R_{x}^{2}+R_{y}^{2}+R_{z}^{2}}$. In particular, if there is no discretization in the $z$ direction, such as in a thin film, then we have $\mathbb{K}_{x z}=\mathbb{K}_{z x}=\mathbb{K}_{y z}=\mathbb{K}_{z y}=0$. The stray field can be calculated using the FFT, see [28, 48] for more details.

\section{Stability analysis of Algorithm 1}

In this subsection, we consider the case $\overline{\mathbf{h}}=0$ and homogeneous Neumann boundary conditions that are dominant in numerical experiments. We will show that the discrete exchange energy decreases in time under certain conditions on the computational mesh for both explicit and implicit mimetic finite difference schemes. More precisely, all matrices $\mathbb{M}_{\mathcal{F}, E}^{-1}$ must be M-matrices. In addition, for the explicit scheme, the time step and the mesh size must satisfy a Courant condition.

Let $\|\cdot\|_{\mathcal{F}}$ and $\|\cdot\|_{\mathcal{F}, E}$ denote the norms indexed by global and local inner products. First, we formulate our main result and then prove it as well as two auxiliary lemmas.

Theorem 1. Let conditions of Lemmas 1 and 2 hold true. For the explicit scheme $(\theta=0)$, we further assume the Courant condition

$$
\frac{k}{h^{2}} \leq \frac{2 \alpha}{C_{1}\left(1+\alpha^{2}\right)}
$$

Then, for any time moment $t^{j}$, we have the following energy estimate:

$$
\| \text { GRAD } \mathbf{m}^{j+1}\left\|_{\mathcal{F}} \leq\right\| \text { GRAD } \mathbf{m}^{j} \|_{\mathcal{F}}
$$


Proof. Let $\hat{\mathbf{m}}^{j+1}$ denote the magnetization solution obtained on step 2a of Algorithm 1. Let $\mathbf{v}^{j}$ denote the change of magnetization, i.e. $\hat{\mathbf{m}}^{j+1}=\mathbf{m}^{j}+k \mathbf{v}^{j}$. Note that $\mathbf{m}_{E}^{j} \cdot \mathbf{v}_{E}^{j}=0$ and $\left|\mathbf{m}_{E}^{j}\right|=1$ for all $E$. Hence, $\left|\hat{\mathbf{m}}_{E}^{j}\right| \geq 1$ and Lemma 2 gives us energy decrease after normalization:

$$
\| \text { GRAD } \mathbf{m}^{j+1}\left\|_{\mathcal{F}} \leq\right\| \operatorname{GRAD} \hat{\mathbf{m}}^{j+1} \|_{\mathcal{F}}
$$

We have the following identity:

$$
\begin{aligned}
\left\|\mathbf{G R A D}\left(\mathbf{m}^{j}+k \mathbf{v}^{j}\right)\right\|_{\mathcal{F}}^{2} & =\left\|\mathbf{G R A D} \mathbf{m}^{j}\right\|_{\mathcal{F}}^{2} \\
& +2 k\left[\mathbf{G R A D} \mathbf{m}^{j}, \mathbf{G R A D ~ v}^{j}\right]_{\mathcal{F}}+k^{2}\left\|\mathbf{G R A D ~ v}^{j}\right\|_{\mathcal{F}}^{2}
\end{aligned}
$$

The second equation in the $\theta$-scheme $(3.13)$ gives us the definition of $\mathbf{v}^{j}$ :

$$
\mathbf{v}_{E}^{j}=-\mathbf{m}_{E}^{j} \times \mathbf{D I V}_{E} \mathbf{p}_{E}^{j+\theta}-\alpha\left(\mathbf{m}_{E}^{j} \cdot \mathbf{D I V}_{E} \mathbf{p}_{E}^{j+\theta}\right) \mathbf{m}_{E}^{j}+\alpha \mathbf{D I V}_{E} \mathbf{p}_{E}^{j+\theta}
$$

Using this formula and the fact that $\left|\mathbf{m}_{E}^{j}\right|=1$, we can calculate the following quantity:

$$
\alpha \mathbf{v}_{E}^{j}+\mathbf{m}_{E}^{j} \times \mathbf{v}_{E}^{j}=-\left(1+\alpha^{2}\right)\left(\left(\mathbf{m}_{E}^{j} \cdot \mathbf{D} \mathbf{I} \mathbf{V}_{E} \mathbf{p}_{E}^{j+\theta}\right) \mathbf{m}_{E}^{j}-\mathbf{D I V}_{E} \mathbf{p}_{E}^{j+\theta}\right) .
$$

Taking the dot product of both sides with $\mathbf{v}_{E}^{j}$, summing up the results weighted with element volumes, and using the duality property of the mimetic operators, we obtain

$$
\begin{aligned}
-\frac{\alpha}{1+\alpha^{2}}\left[\mathbf{v}^{j}, \mathbf{v}^{j}\right]_{\mathcal{Q}} & =-\left[\mathbf{D I V} \mathbf{p}^{j+\theta}, \mathbf{v}^{j}\right]_{\mathcal{Q}}=\left[\operatorname{GRAD}\left(\mathbf{m}^{j}+\theta k \mathbf{v}^{j}\right), \mathbf{G R A D v}^{j}\right]_{\mathcal{Q}} \\
& =\left[\operatorname{GRAD~}^{j}, \mathbf{G R A D v}^{j}\right]_{\mathcal{Q}}+\theta k\left[\operatorname{GRADv}^{j}, \operatorname{GRAD~v}^{j}\right]_{\mathcal{Q}}
\end{aligned}
$$

Inserting 4.5 into 4.3), we have

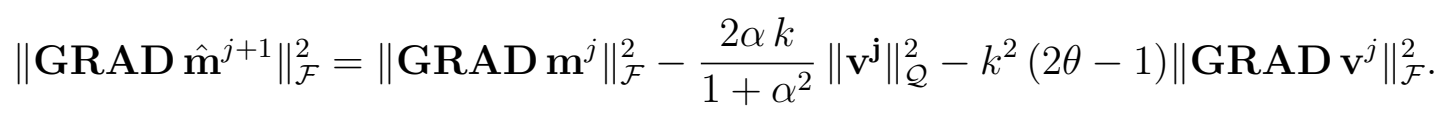

This shows that for the implicit scheme, $\theta=1$, the energy decreases using equation (4.2). For the explicit scheme, we use Lemma 1 to obtain

$$
\left\|\mathbf{G R A D} \mathbf{m}^{j+1}\right\|_{\mathcal{F}}^{2} \leq\left\|\mathbf{G R A D} \mathbf{m}^{j}\right\|_{\mathcal{F}}^{2}-k\left(\frac{2 \alpha}{1+\alpha^{2}}-\frac{C_{1} k}{h^{2}}\right)\left\|\mathbf{v}^{j}\right\|_{\mathcal{Q}^{h}}^{2}
$$

which shows that the energy decreases under the Courant condition 4.1 .

What is left is to prove two technical lemmas used in Theorem 1. The first lemma uses the stability condition of the mimetic scheme. The second lemma assumes that the family of elemental matrices $\mathbb{M}_{\mathcal{F}, E}^{-1}$ contains M-matrices which imposes certain constraints on the shape of mesh cells.

Lemma 1. Let mesh $\Omega^{h}$ be shape regular and quasi-uniform. Then, for any $\mathbf{v}^{h} \in\left(\mathcal{Q}^{h}\right)^{3}$, we have the following inverse estimate:

$$
\left\|\mathbf{G R A D} \mathbf{v}^{h}\right\|_{\mathcal{F}}^{2} \leq \frac{C_{1}}{h^{2}}\left\|\mathbf{v}^{h}\right\|_{\mathcal{Q}}^{2}
$$

where $C_{1}$ is a positive constant independent of $h$ and $\mathbf{v}^{h}$. 
Proof. For each component $u \in\{x, y, z\}$, we use definition of the mimetic operators, and stability condition 3.22 to obtain

$$
\left\|\mathcal{G} \mathcal{R} \mathcal{A} \mathcal{D} v_{u}^{h}\right\|_{\mathcal{F}}=\left\|\mathbb{M}_{\mathcal{F}}^{-1 / 2} \mathcal{D} \mathcal{I} \mathcal{V}^{T} \mathbb{M}_{\mathcal{Q}} v_{u}^{h}\right\| \leq \frac{C_{2}}{h^{d / 2}}\left\|\mathcal{D} \mathcal{I} \mathcal{V}^{T} \mathbb{M}_{\mathcal{Q}} v_{u}^{h}\right\| \leq \frac{C_{2}}{h^{d / 2}}\left\|\mathcal{D} \mathcal{I} \mathcal{V}^{T}\right\|\left\|\mathbb{M}_{\mathcal{Q}} v_{u}^{h}\right\|
$$

where $C_{2}$ depends on $c_{0}$ in 3.22 and the shape regularity of mesh $\Omega^{h}$. The definition of the divergence operator gives $\left\|\mathcal{D} \mathcal{I} \mathcal{V}^{T}\right\| \leq C_{3} h^{-1}$, where $C_{3}$ depends only on the mesh shape regularity constants. The definition of the inner product matrix gives $\left\|\mathbb{M}_{\mathcal{Q}}^{1 / 2}\right\| \leq h^{d / 2}$. Thus, we have

$$
\left\|\mathcal{G} \mathcal{R} \mathcal{A D} v_{u}^{h}\right\|_{\mathcal{F}} \leq \frac{C_{2} C_{3}}{h}\left\|v_{u}^{h}\right\|_{\mathcal{Q}}
$$

The assertion of the lemma follows with $C_{1}=\left(C_{2} C_{3}\right)^{2}$.

Lemma 2. Let each elemental matrix $\mathbb{M}_{\mathcal{F}, E}$ satisfy two conditions: (a) $\mathbb{M}_{\mathcal{F}, E}^{-1}$ is an $M$-matrix, and (b) vector $\mathbb{M}_{\mathcal{F}, E}^{-1} \mathbb{C}_{E} \boldsymbol{e}$ has positive entries. Furthermore, let $\hat{\mathbf{v}}^{h} \in\left(\mathcal{Q}^{h}\right)^{3}$ be any vector and $\mathbf{v}^{h}$ be its normalization such that $\mathbf{v}_{E}=\hat{\mathbf{v}}_{E} /\left|\hat{\mathbf{v}}_{E}\right|$ for all $E$ in $\Omega^{h}$. Finally, let $\left|\hat{\mathbf{v}}_{E}\right| \geq 1$. Then, we have energy decrease after the renormalization:

$$
\left\|\mathbf{G R A D} \mathbf{v}^{h}\right\|_{\mathcal{F}} \leq\left\|\mathbf{G R A D} \hat{\mathbf{v}}^{h}\right\|_{\mathcal{F}}
$$

Proof. Using the global and local mimetic gradient operators, we define vectors

$$
\mathbf{q}=\mathbf{G R A D}^{h} \text { and } \mathbf{q}_{E}=\operatorname{GRAD}_{E}\left(\begin{array}{c}
\mathbf{v}_{E} \\
\widetilde{\mathbf{v}}_{E}
\end{array}\right)
$$

The global and local gradient operators are equivalent when $q_{u, E_{1}, f}+q_{u, E_{2}, f}=0$ on each internal mesh edge shared by two elements $E_{1}$ and $E_{2}$. Under this continuity condition, the additivity property of the inner product gives:

$$
\|\mathbf{q}\|_{\mathcal{F}}^{2}=\sum_{E \in \Omega^{h}}\left\|\mathbf{q}_{E}\right\|_{\mathcal{F}, E}^{2}
$$

Formula (3.11) for the local mimetic gradient operator and definition of diagonal matrix $\mathbb{C}_{E}$ give

$$
\left\|\mathbf{q}_{u, E}\right\|_{\mathcal{F}, E}^{2}=\left(\begin{array}{c}
v_{u, E} \\
\widetilde{v}_{u, E}
\end{array}\right)^{T}\left(\begin{array}{cc}
\boldsymbol{e}^{T} \mathbb{T}_{E} \boldsymbol{e} & -\boldsymbol{e}^{T} \mathbb{T}_{E} \\
-\mathbb{T}_{E} \mathbf{e} & \mathbb{T}_{E}
\end{array}\right)\left(\begin{array}{c}
v_{u, E} \\
\widetilde{v}_{u, E}
\end{array}\right), \quad \mathbb{T}_{E}=\mathbb{C}_{E} \mathbb{M}_{\mathcal{F}, E}^{-1} \mathbb{C}_{E}
$$

The entries of vector $\widetilde{v}_{u, E}^{h}$ are associated with mesh edges and defined completely by the flux continuity condition $q_{u, E_{1}, f}+q_{u, E_{2}, f}=0$. To find another form of this condition, we sum up equations 4.8 which gives

$$
\left\|\mathbf{q}_{u}\right\|_{\mathcal{F}}^{2}=\sum_{E \in \Omega^{h}}\left\|\mathbf{q}_{u, E}\right\|_{\mathcal{F}, E}^{2}=\left(\begin{array}{c}
v_{u}^{h} \\
\widetilde{v}_{u}^{h}
\end{array}\right)^{T}\left(\begin{array}{cc}
\mathbb{T}^{E E} & \mathbb{T}^{E f} \\
\mathbb{T}^{f E} & \mathbb{T}^{f f}
\end{array}\right)\left(\begin{array}{c}
v_{u}^{h} \\
\widetilde{v}_{u}^{h}
\end{array}\right)
$$

Now, inserting (3.11) in the flux continuity equations pre-multiplied by $|f|$, we obtain

$$
\mathbb{T}^{f E} v_{u}^{h}+\mathbb{T}^{f f} \widetilde{v}_{u}^{h}=0 .
$$


Using this relationship in formula (4.9), we obtain

$$
\left\|\mathbf{q}_{u}\right\|_{\mathcal{F}}^{2}=\left(v_{u}^{h}\right)^{T}\left(\mathbb{T}^{E E}-\mathbb{T}^{E f}\left(\mathbb{T}^{f f}\right)^{-1} \mathbb{T}^{f E}\right) v_{u}^{h}=:\left(v_{u}^{h}\right)^{T} \mathbb{S}^{E E} v_{u}^{h}
$$

The Schur complement $\mathbb{S}^{E E}$ has one important property. According to [38], the conditions (a) and (b) imply that the local matrices in (4.8) are singular irreducible M-matrices with the single null vector $\boldsymbol{e}$. Hence, the assembled matrix in 4.9 is a singular M-matrix. From linear algebra we know that the Schur complement is also a singular M-matrix. Since $\mathbb{S}^{E E} \boldsymbol{e}=0$, the following vector-matrix-vector product can be broken into assembly of $2 \times 2$ matrices,

$$
\left(v_{u}^{h}\right)^{T} \mathbb{S}^{E E} v_{u}^{h}=\sum_{i<j} \beta_{i j}\left(\begin{array}{c}
v_{u, E_{i}} \\
v_{u, E_{j}}
\end{array}\right)^{T}\left(\begin{array}{cc}
1 & -1 \\
-1 & 1
\end{array}\right)\left(\begin{array}{c}
v_{u, E_{i}} \\
v_{u, E_{j}}
\end{array}\right)
$$

with non-negative weights $\beta_{i j}$. Recall that $v_{u, E_{i}}=\hat{v}_{u, E_{i}} /\left|\hat{\mathbf{v}}_{E_{i}}\right|$ and $\left|\hat{\mathbf{v}}_{E_{i}}\right| \geq 1$. Thus, we have the following estimate:

$$
\begin{aligned}
\sum_{u \in\{x, y, z\}} & \left(\frac{\left(\hat{v}_{u, E_{i}}\right)^{2}}{\left|\hat{\mathbf{v}}_{E_{i}}\right|^{2}}-2 \frac{\hat{v}_{u, E_{i}}}{\left|\hat{\mathbf{v}}_{E_{i}}\right|} \frac{\hat{v}_{u, E_{j}}}{\left|\hat{\mathbf{v}}_{E_{j}}\right|}+\frac{\left(\hat{v}_{u, E_{j}}\right)^{2}}{\left|\hat{\mathbf{v}}_{E_{j}}\right|^{2}}\right)=2-2 \sum_{u \in\{x, y, z\}} \frac{\hat{v}_{u, E_{i}}}{\left|\hat{\mathbf{v}}_{E_{i}}\right|} \frac{\hat{v}_{u, E_{j}}}{\left|\hat{\mathbf{v}}_{E_{j}}\right|} \\
& \leq\left|\hat{\mathbf{v}}_{E_{i}}\right|\left|\hat{\mathbf{v}}_{E_{j}}\right|\left(\frac{\left|\hat{\mathbf{v}}_{E_{i}}\right|}{\left|\hat{\mathbf{v}}_{E_{j}}\right|}+\frac{\left|\hat{\mathbf{v}}_{E_{j}}\right|}{\left|\hat{\mathbf{v}}_{E_{i}}\right|}-2 \sum_{u \in\{x, y, z\}} \frac{\hat{v}_{u, E_{i}}}{\left|\hat{\mathbf{v}}_{E_{i}}\right|} \frac{\hat{v}_{u, E_{j}}}{\left|\hat{\mathbf{v}}_{E_{j}}\right|}\right)=\sum_{u \in\{x, y, z\}}\left(\hat{v}_{u, E_{i}}-\hat{v}_{u, E_{j}}\right)^{2} .
\end{aligned}
$$

We conclude that

$$
\sum_{u \in\{x, y, z\}}\left(v_{u}^{h}\right)^{T} \mathbb{S}^{E E} v_{u}^{h} \leq \sum_{u \in\{x, y, z\}}\left(\hat{v}_{u}^{h}\right)^{T} \mathbb{S}^{E E} \hat{v}_{u}^{h}=\sum_{u \in\{x, y, z\}}\left\|\mathcal{G} \mathcal{R} \mathcal{A D} \hat{v}_{u}^{h}\right\|_{\mathcal{F}}^{2}
$$

and the assertion of the lemma follows.

Remark 2. To comply with the conditions of Lemma 2, we have to select special matrices $\tilde{\mathbb{G}}$ in (3.24). A simple optimization algorithm for this task is proposed in [36].

\section{Numerical Examples}

\subsection{Explicit time integration scheme $(\theta=0)$}

In this subsection, we consider the explicit time integration scheme, i.e. $\theta=0$ in (3.13). Analytical solutions for the LL equation are available for special forms of the effective field (2.2), for instance when it has only the exchange energy term, $\mathbf{h}=\Delta \mathbf{m}$. We consider the analytical solution from [22] with the periodic boundary conditions, namely

$$
\begin{aligned}
& m_{x}\left(x_{1}, x_{2}, t\right)=\frac{1}{d(t)} \sin \beta \cos \left(\kappa\left(x_{1}+x_{2}\right)+g(t)\right) \\
& m_{y}\left(x_{1}, x_{2}, t\right)=\frac{1}{d(t)} \sin \beta \sin \left(\kappa\left(x_{1}+x_{2}\right)+g(t)\right) \\
& m_{z}\left(x_{1}, x_{2}, t\right)=\frac{1}{d(t)} e^{2 \kappa^{2} \alpha t} \cos \beta
\end{aligned}
$$




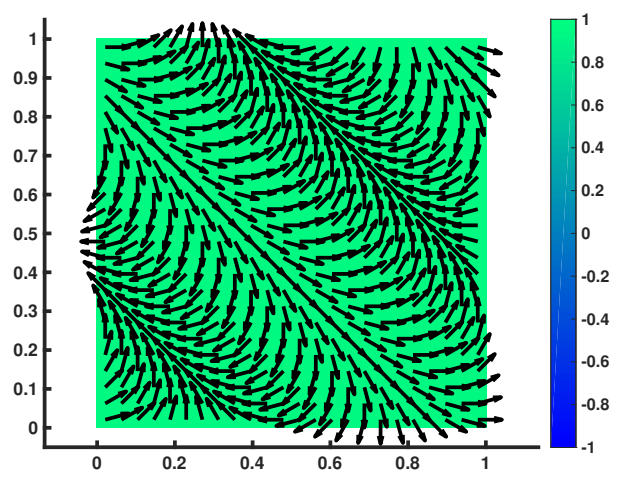

Figure 5.1: Analytical solution 5.1 at time 0. The vectors in the plot denote the $m_{x}$ and $m_{y}$ components, and the color denotes the $m_{z}$ component.

where $\beta=\frac{\pi}{12}, \kappa=2 \pi, d(t)=\sqrt{\sin ^{2} \beta+e^{4 \kappa^{2} \alpha t} \cos ^{2} \beta}$ and $g(t)=\frac{1}{\alpha} \log \left(\frac{d(t)+e^{2 \kappa^{2} \alpha t} \cos \beta}{1+\cos \beta}\right)$. Note that $m_{z} \rightarrow 1$ as $t \rightarrow \infty$. The snapshot of the analytical solution (5.1) at time 0 is in Fig. 5.1. We perform simulation on the time interval $(0, T)$, where $T=0.001$.

Let us consider a square mesh with mesh step $h$ occupying the unit square $\Omega$ and set the time step $k=8 \times 10^{-7} h^{2}$. We measure errors in the magnetization and its flux in the mesh dependent $L^{2}$-type norms $\|\cdot\|_{\mathcal{Q}}$ and $\|\cdot\|_{\mathcal{F}}$, respectively. In addition to that, we consider the maximum norm

$$
\left\|\mathbf{m}^{\mathbf{h}}-\mathbf{m}^{I}\right\|_{L^{\infty}}=\max _{E \in \Omega^{h}}\left|\mathbf{m}_{E}-\mathbf{m}_{E}^{I}\right|,
$$

where $\mathbf{m}^{I} \in\left(\mathcal{Q}^{h}\right)^{3}$ is a projection of the analytical solution on the discrete space. For this projection, we simply take the value of $\mathbf{m}$ at centroids of elements $E$. The projection $\mathbf{p}^{I}$ is defined in a similar way. Table 5.1 shows convergence rates in different norms. Observe that the explicit scheme leads to the second-order convergence for both the magnetization and its flux.

\begin{tabular}{c|c|c|c}
$1 / h$ & $\left\|\mathbf{m}^{h}-\mathbf{m}^{I}\right\|_{L^{\infty}}$ & $\left\|\mathbf{m}^{h}-\mathbf{m}^{I}\right\|_{\mathcal{Q}}$ & $\left\|\mathbf{p}^{h}-\mathbf{p}^{I}\right\|_{\mathcal{F}}$ \\
\hline 32 & $8.222 \mathrm{e}-05$ & $8.360 \mathrm{e}-05$ & $2.967 \mathrm{e}-03$ \\
64 & $2.060 \mathrm{e}-05$ & $2.092 \mathrm{e}-05$ & $7.418 \mathrm{e}-04$ \\
128 & $5.154 \mathrm{e}-06$ & $5.231 \mathrm{e}-06$ & $1.854 \mathrm{e}-04$ \\
256 & $1.289 \mathrm{e}-06$ & $1.308 \mathrm{e}-06$ & $4.636 \mathrm{e}-05$ \\
\hline rate & 2.00 & 2.00 & 2.00
\end{tabular}

Table 5.1: Convergence analysis of the explicit time integration scheme on uniform square meshes.

\subsection{Implicit time integration scheme $(\theta=1)$}

\subsubsection{Uniform square meshes}

In this subsection, we consider the implicit time integration scheme, i.e. $\theta=1$ in $(3.13)$. The analytical solution is given by (5.1). We set the time step $k=0.008 h^{2}$ so that the first-order time integration error will not affect our conclusions. Convergence rates shown in Table 5.2 indicate the second-order convergence for the magnetization and the first-order 
convergence for its flux. A rigorous convergence analysis of Algorithm 1, which is beyond the scope of this work, is required to explain lack of flux super-convergence in this scheme.

\begin{tabular}{c|c|c|c}
$1 / h$ & $\left\|\mathbf{m}^{h}-\mathbf{m}^{I}\right\|_{L^{\infty}}$ & $\left\|\mathbf{m}^{h}-\mathbf{m}^{I}\right\|_{\mathcal{Q}}$ & $\left\|\mathbf{p}^{h}-\mathbf{p}^{I}\right\|_{\mathcal{F}}$ \\
\hline 32 & $9.082 \mathrm{e}-05$ & $9.195 \mathrm{e}-05$ & $2.531 \mathrm{e}-02$ \\
64 & $2.273 \mathrm{e}-05$ & $2.302 \mathrm{e}-05$ & $1.261 \mathrm{e}-02$ \\
128 & $5.687 \mathrm{e}-06$ & $5.756 \mathrm{e}-06$ & $6.301 \mathrm{e}-03$ \\
256 & $1.422 \mathrm{e}-06$ & $1.439 \mathrm{e}-06$ & $3.150 \mathrm{e}-03$ \\
\hline rate & 2.00 & 2.00 & 1.00
\end{tabular}

Table 5.2: Convergence analysis of the implicit time integration scheme on uniform square meshes.

\subsubsection{Smoothly distorted and randomized quadrilateral meshes}

In this subsection, we continue convergence analysis of the implicit time integration scheme using the analytical solution described above. This time, we consider the randomized and smoothly distorted meshes shown in Fig. 5.2. The randomized mesh is built from the uniform square mesh by a random distortion of its nodes:

$$
x:=x+0.2 \xi_{x} h, \quad y:=x+0.2 \xi_{y} h,
$$

where $\xi_{x}$ and $\xi_{y}$ are random variables between -1 and 1 . The mesh perturbation was modified on the boundary so that periodic boundary conditions could be used.

The smoothly distorted mesh is built from the uniform square mesh using a smooth map to calculate new positions of mesh nodes:

$$
\begin{aligned}
& x:=x+0.1 \sin (2 \pi x) \sin (2 \pi y), \\
& y:=y+0.1 \sin (2 \pi x) \sin (2 \pi y) .
\end{aligned}
$$
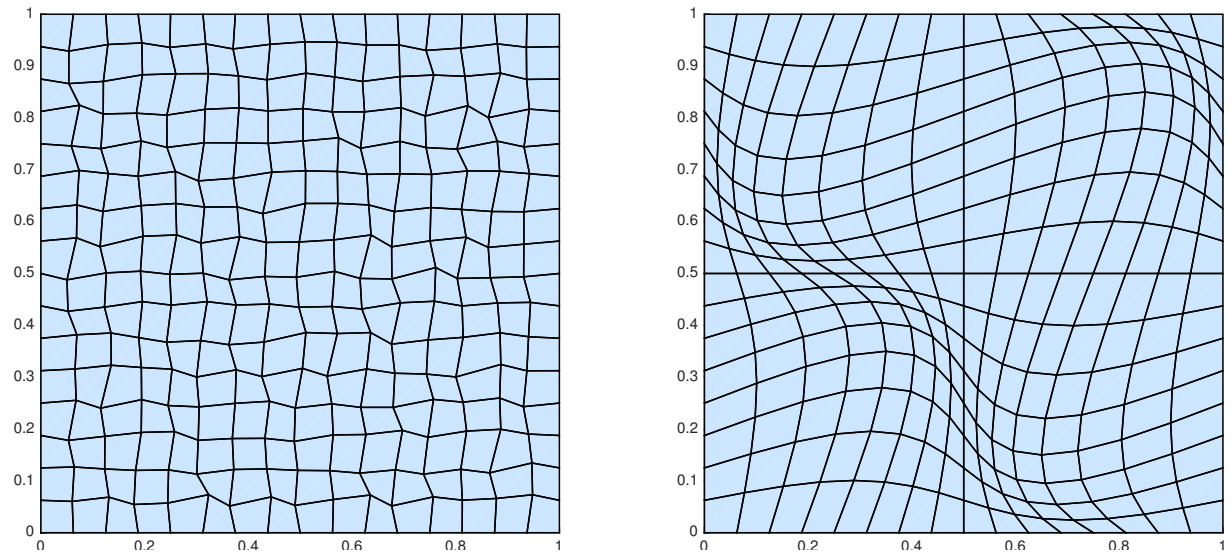

Figure 5.2: Modified meshes: randomized mesh (left), and smoothly distorted mesh (right).

We set the time step $k=0.008 h^{2}$. The errors are summarized in Table 5.3 and Fig. 5.3 . Again, we observe the second-order convergence rate for the magnetization and the first-order convergence rate for its flux. 


\begin{tabular}{c|c|c|c||c|c|c} 
& \multicolumn{3}{|c||}{ Randomized mesh } & \multicolumn{3}{c}{ Smoothly distorted mesh } \\
\hline $1 / h$ & $\left\|\mathbf{m}^{h}-\mathbf{m}^{I}\right\|_{L^{\infty}}$ & $\left\|\mathbf{m}^{h}-\mathbf{m}^{I}\right\|_{\mathcal{Q}}$ & $\left\|\mathbf{p}^{h}-\mathbf{p}^{I}\right\|_{\mathcal{F}}$ & $\left\|\mathbf{m}^{h}-\mathbf{m}^{I}\right\|_{L^{\infty}}$ & $\left\|\mathbf{m}^{h}-\mathbf{m}^{I}\right\|_{\mathcal{Q}}$ & $\left\|\mathbf{p}^{h}-\mathbf{p}^{I}\right\|_{\mathcal{F}}$ \\
\hline 16 & $3.121 \mathrm{e}-03$ & $9.460 \mathrm{e}-04$ & $6.560 \mathrm{e}-02$ & $1.751 \mathrm{e}-03$ & $1.009 \mathrm{e}-03$ & $7.611 \mathrm{e}-02$ \\
32 & $1.005 \mathrm{e}-03$ & $2.988 \mathrm{e}-04$ & $3.249 \mathrm{e}-02$ & $5.477 \mathrm{e}-04$ & $2.809 \mathrm{e}-04$ & $3.000 \mathrm{e}-02$ \\
64 & $2.585 \mathrm{e}-04$ & $7.258 \mathrm{e}-05$ & $1.615 \mathrm{e}-02$ & $1.432 \mathrm{e}-04$ & $7.214 \mathrm{e}-05$ & $1.362 \mathrm{e}-02$ \\
128 & $7.939 \mathrm{e}-05$ & $1.807 \mathrm{e}-05$ & $8.135 \mathrm{e}-03$ & $3.608 \mathrm{e}-05$ & $1.816 \mathrm{e}-05$ & $6.623 \mathrm{e}-03$ \\
\hline rate & 1.79 & 1.92 & 1.00 & 1.87 & 1.94 & 1.17
\end{tabular}

Table 5.3: Convergence analysis of the implicit time integration scheme on distorted meshes.
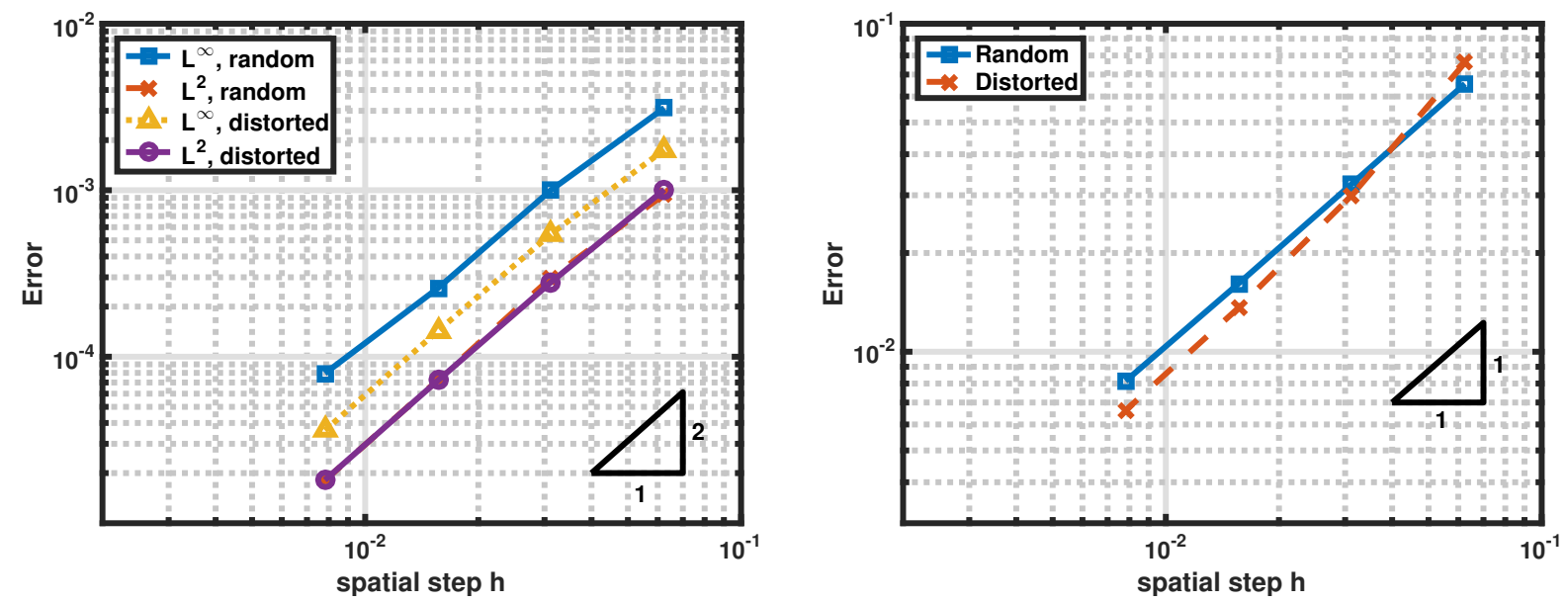

Figure 5.3: Error plot of $\left\|\mathbf{m}^{h}-\mathbf{m}^{I}\right\|_{L^{\infty}}$ and $\left\|\mathbf{m}^{h}-\mathbf{m}^{I}\right\|_{\mathcal{Q}}$ (left) and $\left\|\mathbf{p}^{h}-\mathbf{p}^{I}\right\|_{\mathcal{F}}$ (right) with respect to the mesh size $h$ on the randomized and smoothly distorted meshes shown in Fig. 5.2 .

In these experiments we used formula (3.24) with constant $\tilde{\gamma}$ defined by the scaled trace of the first term, so that $\mathbb{M}_{\mathcal{F}, E}^{-1}$ is not always an M-matrix. Therefore, in Fig. 5.5, we plot the exchange energy $\frac{1}{2}\left[\mathbf{p}^{h}, \mathbf{p}^{h}\right]_{\mathcal{F}}$ as the function of time for two different mesh resolutions $h=\frac{1}{32}$ and $h=\frac{1}{64}$ and for both randomized and smoothly distorted meshes. The figure shows monotone decrease of the exchange energy in time. These results suggest that the M-matrix conditions are sufficient but may not be necessary.

Furthermore, we study the impact of the constant $\tilde{\gamma}$ in $(3.24)$ on the solution accuracy. Fig. 5.4 shows errors as functions of $\gamma_{0}$ on a randomly distorted mesh with mesh size $h=1 / 32$, where $\tilde{\gamma}=\gamma_{0} \frac{1}{|E|} \operatorname{trace}\left(\mathbb{N} \mathbb{N}^{T}\right)$. With only one free parameter, we are not able to minimize errors in both, the magnetization and its flux, and the full matrix of parameter has to be used instead. Also, note that there is much room for varying $\tilde{\gamma}$ with relatively small increase of the error.

\subsubsection{Convergence analysis for problems with the Dirichlet boundary condition}

In this subsection, we conduct numerical experiments for the LL equation with the effective field (2.2) $\boldsymbol{h}=\Delta \mathbf{m}$ using Algorithm 1 and the implicit time integration scheme. We consider the analytical solution (5.1) but now with the Dirichlet boundary condition. The Dirichlet boundary condition allows us to consider more general domains as the circular 


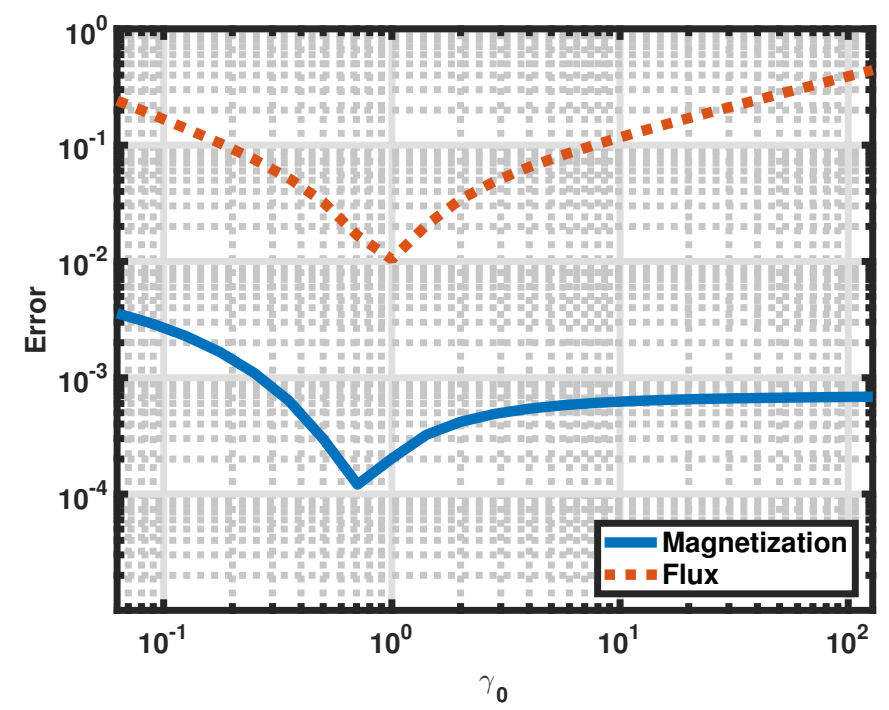

Figure 5.4: Errors as function of $\gamma_{0}$ on a randomly distorted mesh with mesh size $h=1 / 32$ (Fig. 5.2 left)
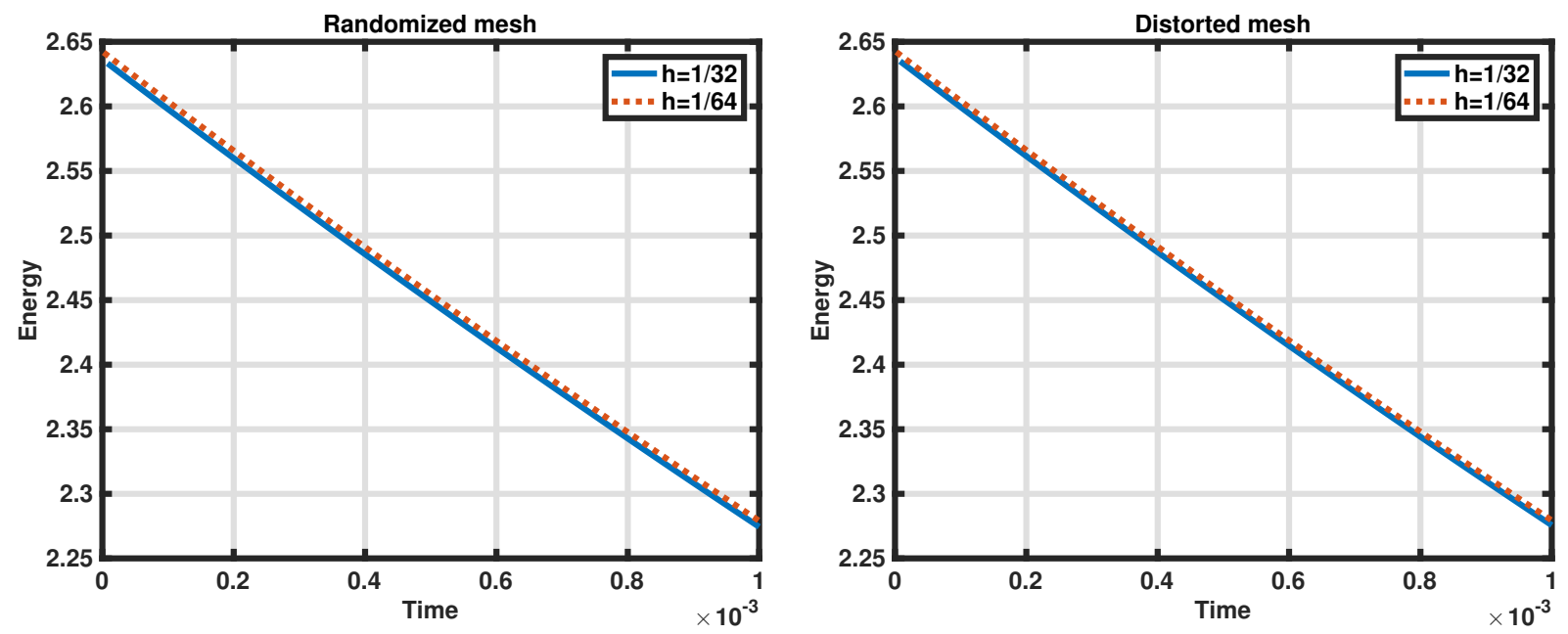

Figure 5.5: Decrease of the exchange energy on randomized (left) and smoothly distorted (right) meshes.

domain with center $(0.5,0.5)$ and radius 0.5 shown on the left panel in Fig. 5.6. This figure shows also a logically square mesh fitted to the domain. This mesh has four elements (corresponding to four corners of the original square mesh) that are almost triangles. However, for the mimetic framework, such elements are classified as shape regular elements (see [12] for more detail) and do not alter the convergence rates.

The computed errors are summarized in Table 5.4 and Fig. 5.7, where we present convergence results for the unit square, circular domain with various meshes. In addition to distorted meshes described in the previous subsection, we conduct numerical experiments on a sequence of polygonal meshes as in Fig. 5.6. The time step is $k=0.008 h^{2}$ for all meshes but polygonal ones where it is set to $k=0.004 h^{2}$. We observe again the second-order 

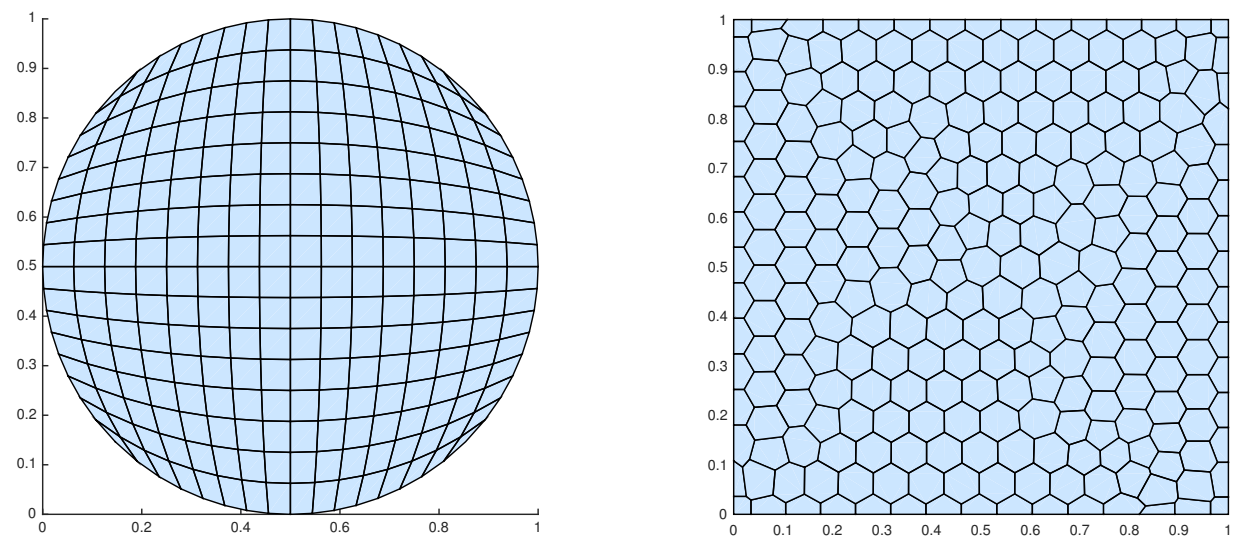

Figure 5.6: Logically square mesh fitted to the circular domain (left panel) and polygonal mesh (right panel).

convergence rate for the magnetization and the first-order convergence rate for its flux.
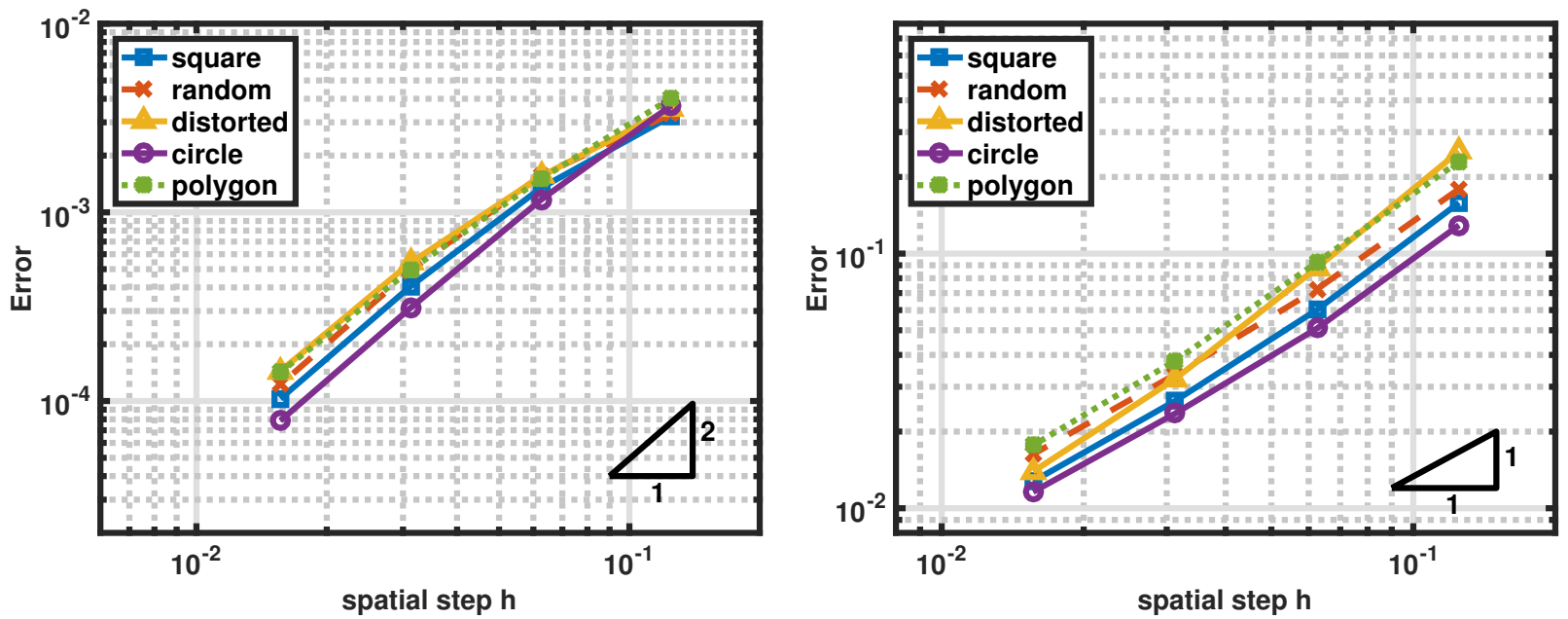

Figure 5.7: Error plot of $\left\|\mathbf{m}^{h}-\mathbf{m}^{I}\right\|_{\mathcal{Q}}$ (left) and $\left\|\mathbf{p}^{h}-\mathbf{p}^{I}\right\|_{\mathcal{F}}$ (right) with respect to the mesh size $h$ for the problem with the Dirichlet boundary conditions. 
Uniform square meshes

\begin{tabular}{|c|c|c|c|c|c|c|}
\hline $1 / h$ & $\left\|\mathbf{m}^{h}-\mathbf{m}^{I}\right\|_{L^{\infty}}$ & ratio & $\left\|\mathbf{m}^{h}-\mathbf{m}^{I}\right\|_{\mathcal{Q}}$ & ratio & $\left\|\mathbf{p}^{h}-\mathbf{p}^{I}\right\|_{\mathcal{F}}$ & ratio \\
\hline 8 & $6.799 \mathrm{e}-03$ & 0.83 & $3.239 \mathrm{e}-03$ & 0.83 & $1.585 \mathrm{e}-01$ & 1.40 \\
\hline 16 & $3.812 \mathrm{e}-03$ & 1.81 & $1.347 \mathrm{e}-03$ & 1.73 & $6.021 \mathrm{e}-02$ & 1.19 \\
\hline 32 & $1.088 \mathrm{e}-03$ & 2.00 & $4.061 \mathrm{e}-04$ & 1.98 & $2.639 \mathrm{e}-02$ & 1.05 \\
\hline 64 & $2.717 \mathrm{e}-04$ & & $1.028 \mathrm{e}-04$ & & $1.275 \mathrm{e}-02$ & \\
\hline & \multicolumn{6}{|c|}{ Randomized meshes } \\
\hline 8 & $6.889 \mathrm{e}-03$ & 0.89 & $3.335 \mathrm{e}-03$ & 1.09 & $1.795 \mathrm{e}-01$ & 1.31 \\
\hline 16 & e-03 & 2 & e-03 & 1.6 & $7.254 \mathrm{e}-02$ & 1.12 \\
\hline 32 & $1.212 \mathrm{e}-03$ & 1.85 & $e-04$ & 2.01 & $3.348 \mathrm{e}-02$ & 1.04 \\
\hline \multirow[t]{2}{*}{64} & $3.370 \mathrm{e}-04$ & & $1.221 \mathrm{e}-04$ & & $1.630 \mathrm{e}-02$ & \\
\hline & \multicolumn{6}{|c|}{ Smoothly distorted meshes } \\
\hline 8 & $6.451 \mathrm{e}-03$ & 0.57 & $3.562 \mathrm{e}-03$ & 1.20 & $2.522 \mathrm{e}-01$ & 1.52 \\
\hline 16 & 49e-03 & 1.49 & e-03 & 1.52 & $8.808 \mathrm{e}-02$ & 1.45 \\
\hline 32 & $1.551 \mathrm{e}-03$ & 1.61 & $5.414 \mathrm{e}-04$ & 1.91 & $3.220 \mathrm{e}-02$ & 1.21 \\
\hline \multirow[t]{2}{*}{64} & $5.073 \mathrm{e}-04$ & & $1.440 \mathrm{e}-04$ & & $1.393 \mathrm{e}-02$ & \\
\hline & \multicolumn{6}{|c|}{$\overline{\text { Polygonal meshes }}$} \\
\hline 8 & 771e-03 & 0.72 & $4.029 \mathrm{e}-03$ & 1.42 & $2.301 \mathrm{e}-01$ & 1.32 \\
\hline 16 & $88 \mathrm{e}-03$ & 1.40 & $1.506 \mathrm{e}-03$ & 1.60 & $9.242 \mathrm{e}-02$ & 1.28 \\
\hline 32 & $23 \mathrm{e}-03$ & 1.71 & $4.970 \mathrm{e}-04$ & 1.81 & $3.794 \mathrm{e}-02$ & 1.10 \\
\hline 64 & $4.957 \mathrm{e}-04$ & 1.85 & $1.422 \mathrm{e}-04$ & 2.28 & $1.765 \mathrm{e}-02$ & 1.06 \\
\hline 128 & $1.372 \mathrm{e}-04$ & & $2.929 \mathrm{e}-05$ & & $8.495 \mathrm{e}-03$ & \\
\hline & \multicolumn{6}{|c|}{ Logically square meshes in the circular domain } \\
\hline 8 & $2.388 \mathrm{e}-02$ & 1.68 & & 1.6 & $1.293 \mathrm{e}-01$ & 1.35 \\
\hline 16 & $\mathrm{e}-03$ & 1.87 & & 1. & e- 02 & 1.11 \\
\hline 32 & $2.032 \mathrm{e}-03$ & 1.95 & $3.120 \mathrm{e}-04$ & 1.99 & $2.354 \mathrm{e}-02$ & 1.02 \\
\hline 64 & $5.268 \mathrm{e}-04$ & & $7.856 \mathrm{e}-05$ & & $1.159 \mathrm{e}-02$ & \\
\hline
\end{tabular}

Table 5.4: Convergence analysis of Algorithm 1 for the case of Dirichlet boundary conditions. 


\subsection{NIST micromag standard problem 4}

The micromag standard problem 4 simulates the magnetization dynamics in a permalloy thin film with two different applied fields [1]. The thin film has dimensions $500 \mathrm{~nm} \times 125 \mathrm{~nm} \times$ 3nm. Before its nondimensionalization, the LL equation [41] reads

$$
\frac{\partial \mathbf{M}}{\partial t^{\prime}}=-\frac{\gamma}{1+\alpha^{2}} \mathbf{M} \times \mathbf{H}-\frac{\gamma \alpha}{M_{s}\left(1+\alpha^{2}\right)} \mathbf{M} \times(\mathbf{M} \times \mathbf{H})
$$

subject to the homogeneous Neumann boundary condition on $\partial \Omega$ and the initial condition described below. Here

$$
\mathbf{H}=-\frac{1}{\mu_{0} M_{s}} \frac{\partial \mathcal{E}}{\partial \mathbf{m}}, \quad \mathcal{E}(\mathbf{M})=\int_{\Omega} \frac{A}{2 M_{s}^{2}}|\nabla \mathbf{M}|^{2}-\mu_{0}\left(\mathbf{H}_{e} \cdot \mathbf{M}\right)-\frac{1}{2} \mu_{0}\left(\mathbf{H}_{s} \cdot \mathbf{M}\right) \mathrm{d} x,
$$

where $\mathbf{M}=M_{s} \mathbf{m}, \mathbf{H}_{e}$ is the external field and $\mathbf{H}_{s}$ is the stray field. Other parameters are the exchange constant $A=2.6 \times 10^{-11}\left[\mathrm{~J} \cdot \mathrm{m}^{-1}\right]$, saturation magnetization $M_{s}=8 \times 10^{5}\left[\mathrm{~A} \cdot \mathrm{m}^{-1}\right]$, gyromagnetic ratio $\gamma=2.21 \times 10^{5}\left[\mathrm{~m} \cdot A^{-1} \cdot \mathrm{s}^{-1}\right]$, magnetic permeability of vacuum $\mu_{0}=$ $4 \pi \times 10^{-7}\left[N \cdot A^{-2}\right]$ and the dimensionless damping parameter $\alpha=0.02$.

By rescaling $\mathbf{H}=M_{s} \mathbf{h}, \mathbf{H}_{s}=M_{s} \mathbf{h}_{s}, \mathbf{H}_{e}=M_{s} \mathbf{h}_{e}, x=L x^{\prime}$ with $L=10^{-9}$, and $t=\frac{1+\alpha^{2}}{\gamma M_{s}} t^{\prime}$, we get equation 2.1 with $\eta=\frac{A}{\mu_{0} M_{s}^{2} L^{2}}$ and equation 2.2 with $Q=0$. The initial state is an equilibrium S-state as in Fig. [5.8 which is obtained by applying an external field of $2 T$ along direction $[1,1,1]$ and slowly reducing it to zero by $0.02 T$ each time step [1, 32].

For a thin film, we may assume that the magnetization is constant in the vertical direction and solve the two-dimensional LL equation. Let us consider a $100 \times 25$ rectangular mesh of square cells with size $h_{x}=h_{y}=5 \mathrm{~nm}$. We use the explicit time integration scheme with time step $\hat{k}=\frac{0.005}{\gamma M_{s}} \approx 28.28$ fs and implicit time discretization scheme with five different time steps $\hat{k}=\frac{0.01}{\gamma M_{s}} \approx 56.56 \mathrm{fs}, \hat{k} \approx 0.14 \mathrm{ps}, \hat{k} \approx 0.28 \mathrm{ps}, \hat{k} \approx 0.57 \mathrm{ps}$, and $\hat{k} \approx 1.13 \mathrm{ps}$.

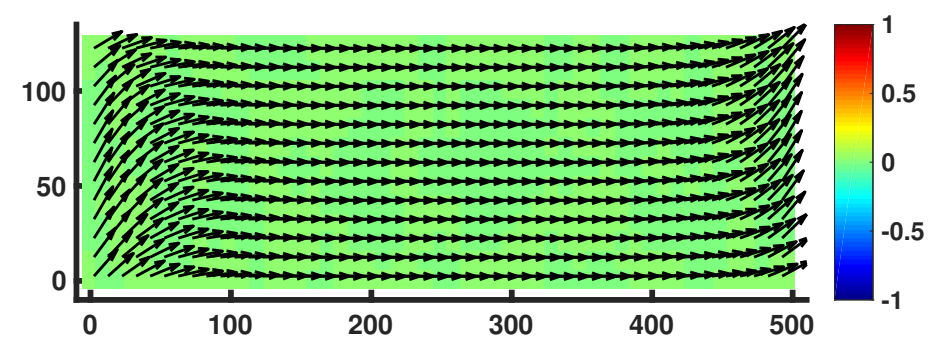

Figure 5.8: NIST 4th problem: the initial equilibrium S-state. The vectors in the plot denote the $m_{x}$ and $m_{y}$ components, and the color denotes the $m_{z}$ component.

The time evolution of the magnetization is simulated using two different applied fields. The first field is $\mu_{0} \mathbf{H}_{e}=(-24.6,4.3,0.0)[\mathrm{mT}]$ which makes angle of approximately 170 degrees with the positive direction of the x-axis. The second field is $\mu_{0} \mathbf{H}_{e}=(-35.5,6.3,0.0)[m T]$ which makes angles of approximately 190 degrees with the positive direction of the x-axis.

The evolution of the average magnetization

$$
\langle\mathbf{m}\rangle=\frac{1}{N_{E}} \sum_{E \in \Omega_{h}} \mathbf{m}_{E}
$$



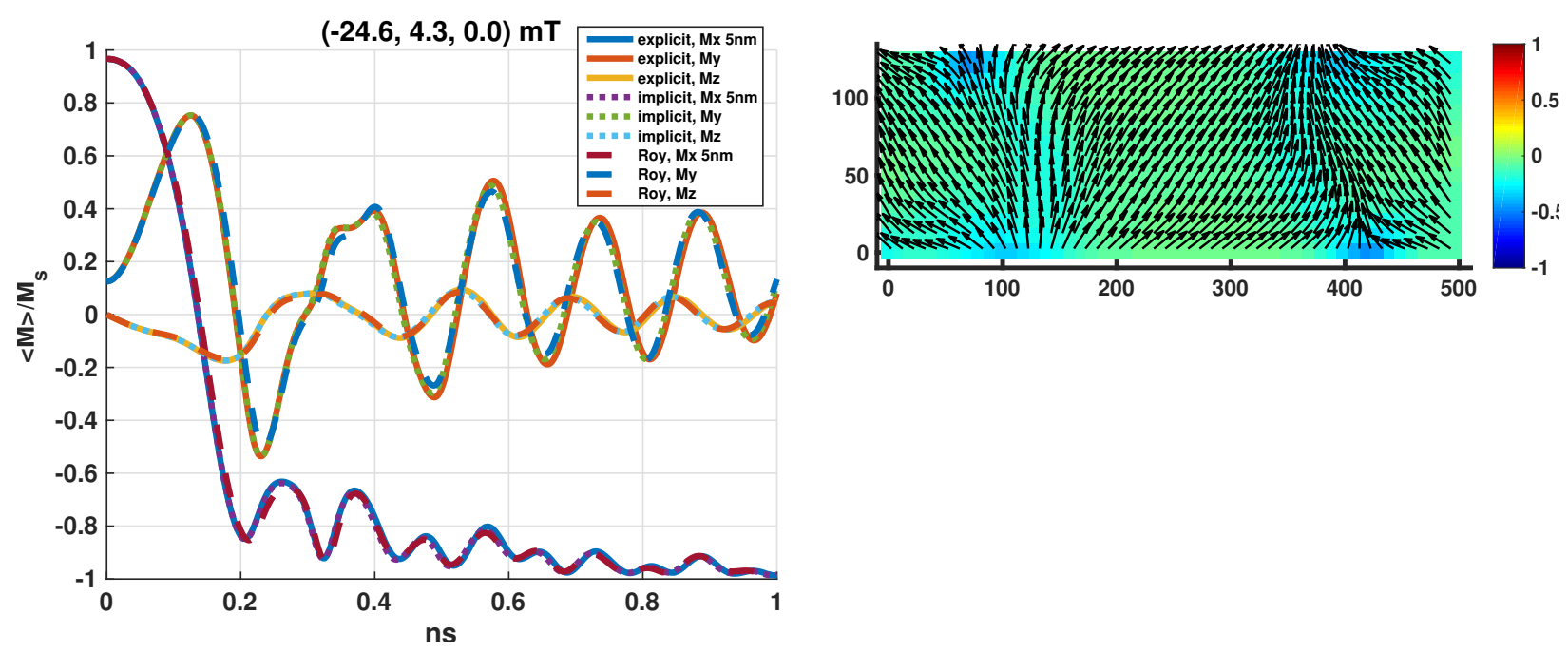

Figure 5.9: NIST 4th problem with the external field $\mu_{0} \mathbf{H}_{e}=(-24.5,4.3,0.0)[\mathrm{mT}]$. Left panel compares the time evolution of the average magnetization calculated using the explicit and implicit mimetic schemes with Roy and Svedlindh's results in [1. Right panel shows the magnetization field when $\left\langle m_{x}\right\rangle$ first crosses zero. The vectors in the plot denote the $m_{x}$ and $m_{y}$ components, and the color denotes the $m_{z}$ component.

with the first field is shown in Fig. 5.9 and compared with the results obtained by Roy and Svedlindh in [1]. They used a finite difference method (leading to the conventional 5-point approximation for the Laplacian) and RK4 for the time stepping with time step $\hat{k} \approx 11$ fs. Also, the magnetization field when $\left\langle m_{x}\right\rangle$ first crosses zero is shown on the right panel in Fig. 5.9. The evolution of the magnetization is qualitatively in a very good agreement.

Consider now the second external field. The evolution of the average magnetization and the magnetization field when $\left\langle m_{x}\right\rangle$ first crosses zero is shown in Fig. 5.10. As mentioned in [1, solutions obtained with different schemes begin to diverge approximately after $0.35 \mathrm{~ns}$. Note that we have a qualitatively good agreement until this time moment.

Furthermore, in Fig. 5.11, the evolution of the magnetization for both applied fields with different time steps is plotted, which shows the stability and temporal convergence of the implicit scheme of Algorithm 1.

\subsection{Domain wall structures in a thin film}

In this subsection, we conduct numerical experiments of the domain wall structures in thin films with no external field using both explicit and implicit time discretization schemes. A similar numerical experiment was conducted in [48] using the Gauss-Seidel projection method and gradually increasing the thickness of the film. Before its nondimensionalization, the LL equation [48] reads

$$
\frac{\partial \mathbf{M}}{\partial t^{\prime}}=-\gamma \mu_{0} \mathbf{M} \times \mathbf{H}-\frac{\gamma \alpha \mu_{0}}{M_{s}} \mathbf{M} \times(\mathbf{M} \times \mathbf{H})
$$

subject to homogeneous Neumann boundary conditions and the initial condition described below. The effective field $\mathbf{H}$ is given by (5.5) but most of the parameters described in that subsection have different values for this experiment. We set the exchange constant $A=2.1 \times 10^{-11}\left[\mathrm{~J} \cdot \mathrm{m}^{-1}\right]$, saturation magnetization $M_{s}=1.71 \times 10^{6}\left[\mathrm{~A} \cdot \mathrm{m}^{-1}\right]$, gyromagnetic 

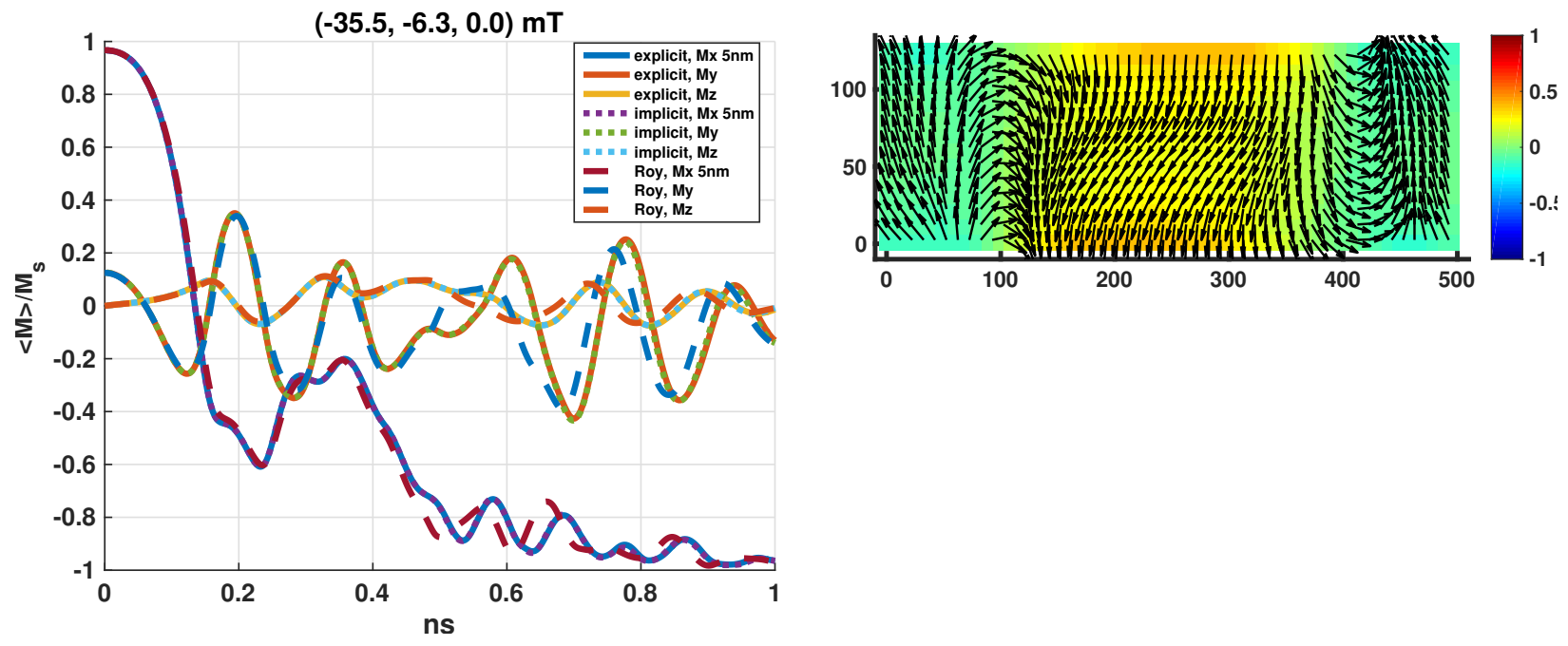

Figure 5.10: NIST 4th problem with the external field $\mu_{0} \mathbf{H}_{e}=(-35.5,6.3,0.0)[m T]$. Left panel compares the time evolution of the average magnetization calculated using the explicit and implicit mimetic schemes with Roy and Svedlindh's results in [1. Right panel shows the magnetization field when $\left\langle m_{x}\right\rangle$ first crosses zero. The vectors in the plot denote the $m_{x}$ and $m_{y}$ components, and the color denotes the $m_{z}$ component.
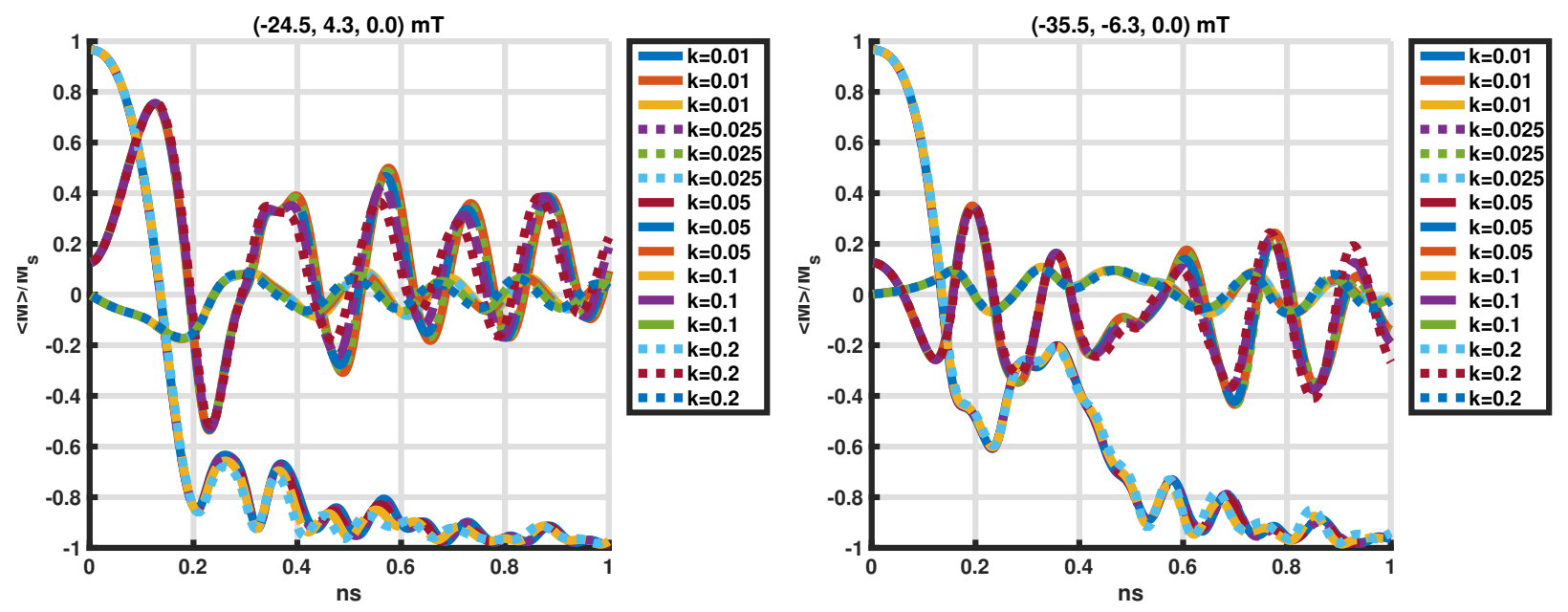

Figure 5.11: NIST 4th problem: evolution of average magnetization computed using Algorithm 1 with various time steps $\hat{k}=\frac{k}{\gamma M_{s}}$ for applied fields $\mu_{0} \mathbf{H}_{e}=(-24.5,4.3,0.0)[m T]$ (left) and $\mu_{0} \mathbf{H}_{e}=(-35.5,6.3,0.0)[m T]$ (right).

ratio $\gamma=1.76 \times 10^{11}\left[T^{-1} \cdot s^{-1}\right]$, magnetic permeability of vacuum $\mu_{0}=4 \pi \times 10^{-7}\left[N \cdot A^{-2}\right]$, and the dimensionless damping parameter $\alpha=0.02$.

Using a slightly different rescaling than above, $\mathbf{H}=M_{s} \mathbf{h}, \mathbf{H}_{s}=M_{s} \mathbf{h}_{s}, \mathbf{H}_{e}=M_{s} \mathbf{h}_{e}$, $x=L x^{\prime}$ with $L=10^{-9}$, and $t=\frac{1}{\mu_{0} \gamma M_{s}} t^{\prime}$, we get equation 2.1) with $\eta=\frac{A}{\mu_{0} M_{s}^{2} L^{2}}$ and equation 2.2 with $Q=0$. We consider a rectangular thin film of size $240 \mathrm{~nm} \times 480 \mathrm{~nm} \times$ $7 \mathrm{~nm}$. Neglecting variation of the magnetization in the vertical direction, we solve the twodimensional LL equation on a $64 \times 128$ mesh of square cells with size $h_{x}=h_{y}=3.75 \mathrm{~nm}$. For the explicit time integration scheme, we use time step $k=\frac{0.01}{\mu_{0} \gamma M_{s}} \approx 26.44 \mathrm{fs}$. For the implicit time discretization scheme, we set $k=\frac{0.25}{\mu_{0} \gamma M_{s}} \approx 0.66 \mathrm{ps}$. The initial state is a 
uniform Néel structure, with $\mathbf{m}=(0,1,0)$ for $0<x<120 \mathrm{~nm}$ and $\mathbf{m}=(0,-1,0)$ for $120 \mathrm{~nm}<x<240 \mathrm{~nm}$ as shown on the left-top panel in Fig. 5.12. The other panels show evolution of the magnetization. We observe that there is a transition from the Néel wall to four $90^{\circ}$ Néel walls connecting a vortex which is the equilibrium state. Note that we plotted the magnetizations on a coarser grid in Fig. 5.12 for better visualization.

For each time step, the computational complexity involves one stray field computation and one linear solver accelerated with an algebraic multigrid preconditioner. The approximate cost for the stray field computation is $O(N \log N)$ and algrebraic multigrid is $O(N)$ using the software library Hypre [19], where $N$ is the number of degrees of freedom. More precisely, the cost for the stray field computation in a thin film is about 8 times the cost of a $2 \mathrm{D}$ FFT. The cost for the algebraic multigrid method is about (complexity +1$) \times \#$ itrs $\times 216 N$, where \#itrs is about 9 and complexity is about 1.67 in our case.

\subsection{Adaptive mesh refinement}

Dynamics of domain walls shows a strong need for adaptive meshes. In this subsection, we compare performance of the MFD method on uniform and locally refined meshes with prescribed structure. Development of a true adaptive algorithm is beyond the scope of this paper. We consider the 1D steady-state solution with the Dirichlet boundary conditions:

$$
\begin{aligned}
& m_{x}\left(x_{1}, x_{2}, t\right)=\sin \left(\phi\left(x_{1}, x_{2}, t\right)\right) \\
& m_{y}\left(x_{1}, x_{2}, t\right)=\cos \left(\phi\left(x_{1}, x_{2}, t\right)\right) \quad \phi\left(x_{1}, x_{2}, t\right)=\pi\left(1+e^{-s \pi\left(x_{1}-b / 2\right)}\right)^{-1}, \\
& m_{z}\left(x_{1}, x_{2}, t\right)=0
\end{aligned}
$$

where $b=1$ and $s=20$. This is a steady-state solution of the LL equation (2.1)-(2.2) with the external field

$$
\begin{aligned}
& \left(\mathbf{h}_{\mathbf{e}}\right)_{x}\left(x_{1}, x_{2}, t\right)=\left(\frac{\partial \phi\left(x_{1}, x_{2}, t\right)}{\partial x}\right)^{2} \sin \left(\phi\left(x_{1}, x_{2}, t\right)\right)-\frac{\partial^{2} \phi\left(x_{1}, x_{2}, t\right)}{\partial x^{2}} \cos \left(\phi\left(x_{1}, x_{2}, t\right)\right) \\
& \left(\mathbf{h}_{\mathbf{e}}\right)_{y}\left(x_{1}, x_{2}, t\right)=\left(\frac{\partial \phi\left(x_{1}, x_{2}, t\right)}{\partial x}\right)^{2} \cos \left(\phi\left(x_{1}, x_{2}, t\right)\right)+\frac{\partial^{2} \phi\left(x_{1}, x_{2}, t\right)}{\partial x^{2}} \sin \left(\phi\left(x_{1}, x_{2}, t\right)\right) \\
& \left(\mathbf{h}_{\mathbf{e}}\right)_{z}\left(x_{1}, x_{2}, t\right)=0
\end{aligned}
$$

$Q=0$ and $\mathbf{h}_{s}=0$. This solution has a sharp transition on interval $0.4<x_{1}<0.6$ and is almost constant on the other regions as shown on the left-top panel in Fig 5.13. The locally refined meshes are polygonal meshes of squares and degenerate pentagons. They are shown on the remaining panels in Fig 5.13 .

The convergence results are summarized in Table 5.5. For about the same numerical cost, the locally refined meshes lead to much more accurate simulations. We expect even better behavior for adaptive meshes built using an error indicator.

\section{Acknowledgements}

This work was carried out under the auspices of the National Nuclear Security Administration of the U.S. Department of Energy at Los Alamos National Laboratory under Contract 
Uniform square meshes

\begin{tabular}{c|c|c|c|c}
\hline Number of cells & $\left\|\mathbf{m}^{h}-\mathbf{m}^{I}\right\|_{L^{\infty}}$ & ratio & $\left\|\mathbf{m}^{h}-\mathbf{m}^{I}\right\|_{\mathcal{Q}}$ & ratio \\
\hline 256 & $9.170 \mathrm{e}-01$ & 1.51 & $3.429 \mathrm{e}-01$ & 1.67 \\
1024 & $3.231 \mathrm{e}-01$ & 2.67 & $1.081 \mathrm{e}-01$ & 2.81 \\
4096 & $5.072 \mathrm{e}-02$ & 2.09 & $1.542 \mathrm{e}-02$ & 2.10 \\
16384 & $1.192 \mathrm{e}-02$ & & $3.605 \mathrm{e}-03$ & \\
\hline \multicolumn{5}{c}{ Adaptive mesh } \\
\hline 220 & $8.993 \mathrm{e}-01$ & 3.52 & $2.906 \mathrm{e}-01$ & 3.60 \\
952 & $6.846 \mathrm{e}-02$ & 3.30 & $2.076 \mathrm{e}-02$ & 3.36 \\
3760 & $7.099 \mathrm{e}-03$ & 1.39 & $2.062 \mathrm{e}-03$ & 1.99 \\
15904 & $2.609 \mathrm{e}-03$ & & $4.915 \mathrm{e}-04$ &
\end{tabular}

Table 5.5: Comparison of errors between uniform and locally refined meshes, see Fig. 5.13 ,

No. DE-AC52-06NA25396. The first author was supported by the U.S. Department of Energy, Office of Science, Office of Workforce Development for Teachers and Scientists, Office of Science Graduate Student Research (SCGSR) program. The SCGSR program is administered by the Oak Ridge Institute for Science and Education for the DOE under contract number DE-AC05-06OR23100. The second author acknowledges the support of the U.S. Department of Energy Office of Science Advanced Scientific Computing Research (ASCR) Program in Applied Mathematics Research.

The authors thank Pieter Swart for useful comments that shaped our selection of numerical experiments. 

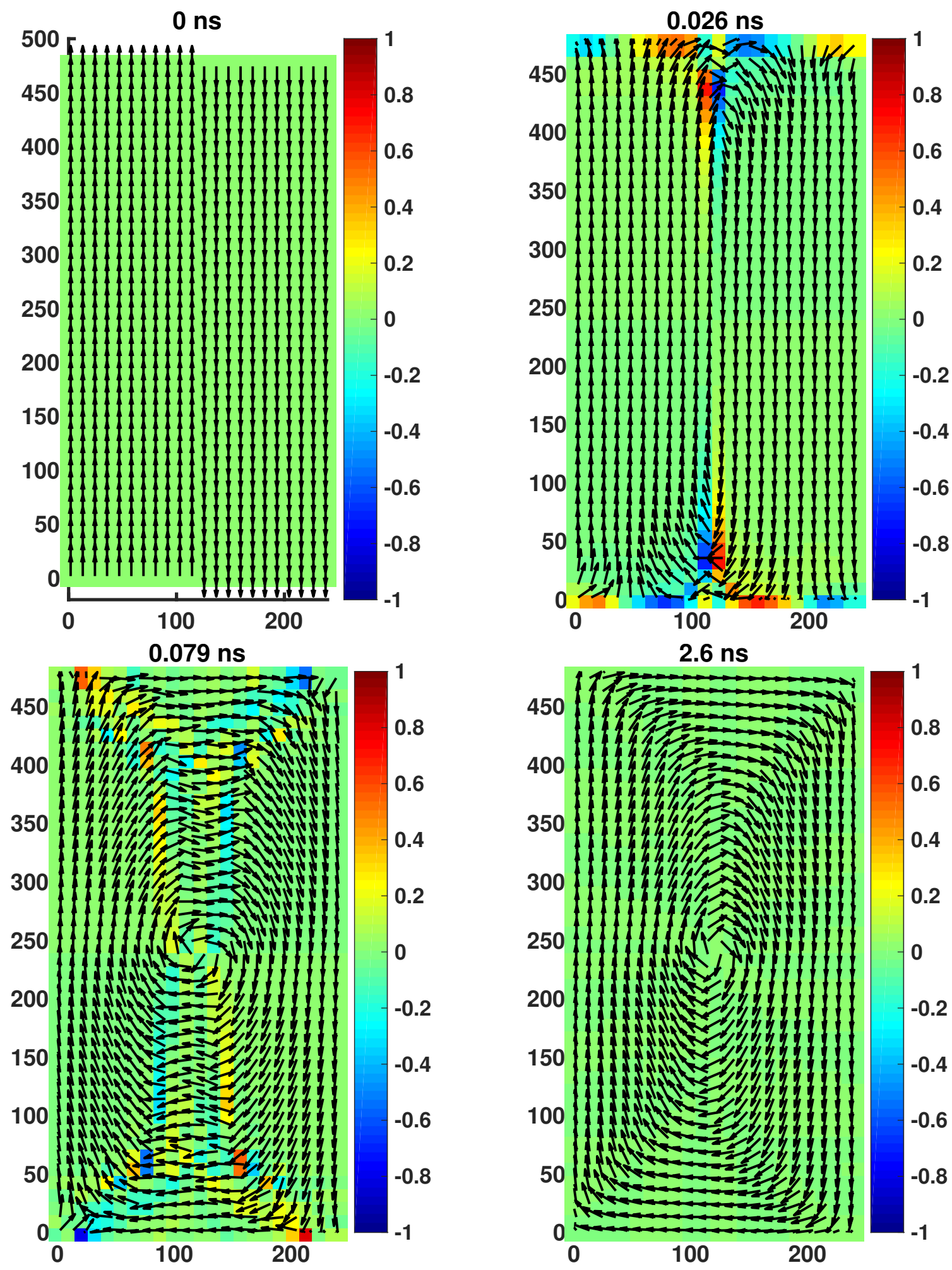

Figure 5.12: Transition from the Néel wall to vortex structure. The vectors in the plot denote the $m_{x}$ and $m_{y}$ components, and the color denotes the $m_{z}$ component. 

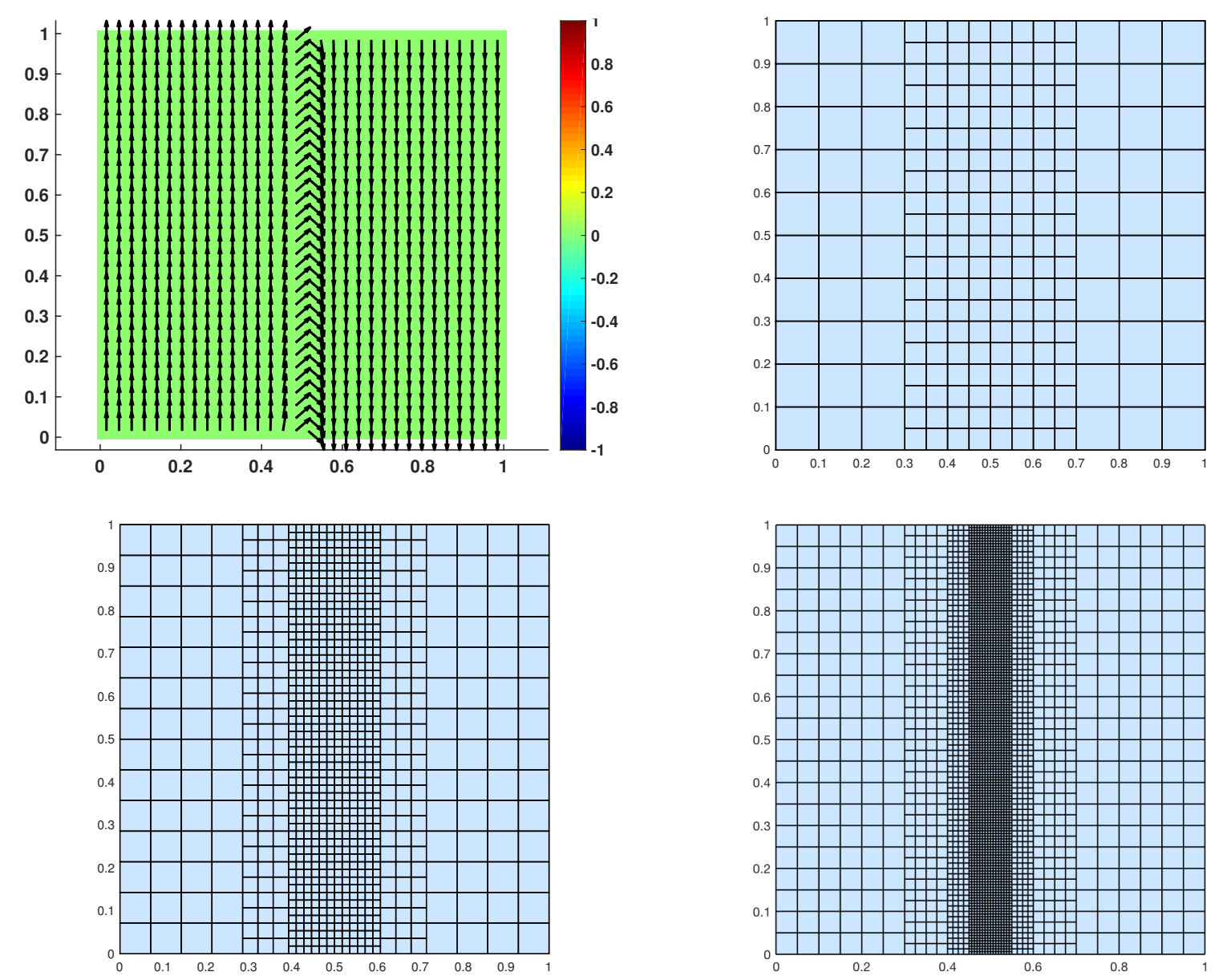

Figure 5.13: Steady state solution on a uniform mesh and three first locally refined meshes. 


\section{References}

[1] $\mu$ MAG Micromagnetic Modeling Activity Group. http://www.ctcms.nist.gov/ rdm/ mumag.org.html.

[2] C. Abert, L. Exl, G. Selke, A. Drews, and T. Schrefl. Numerical methods for the strayfield calculation: A comparison of recently developed algorithms. Journal of Magnetism and Magnetic Materials, 326:176-185, 2013.

[3] C. Abert, G. Selke, B. Krüger, and A. Drews. A fast finite-difference method for micromagnetics using the magnetic scalar potential. IEEE Transactions on Magnetics, 48(3):1105-1109, 2012.

[4] F. Alouges. A new finite element scheme for Landau-Lifchitz equations. Discrete Contin. Dyn. Syst. Ser. S, 1(2):187-196, 2008.

[5] F. Alouges and P. Jaisson. Convergence of a finite element discretization for the LandauLifshitz equations in micromagnetism. Mathematical Models and Methods in Applied Sciences, 16(02):299-316, 2006.

[6] F. Alouges, E. Kritsikis, J. Steiner, and J.-C. Toussaint. A convergent and precise finite element scheme for Landau-Lifschitz-Gilbert equation. Numerische Mathematik, 128(3):407-430, 2014.

[7] F. Alouges, E. Kritsikis, and J.-C. Toussaint. A convergent finite element approximation for Landau-Lifschitz-Gilbert equation. Physica B: Condensed Matter, 407(9):13451349, 2012.

[8] S. Bartels and A. Prohl. Convergence of an implicit finite element method for the Landau-Lifshitz-Gilbert equation. SIAM Journal on Numerical Analysis, 44(4):14051419, 2006.

[9] L. Beirao da Veiga, K. Lipnikov, and G. Manzini. The Mimetic Finite Difference Method for Elliptic PDEs. Springer, 2014. ISBN 978-3-319-02662-6.

[10] G. Bertotti, C. Serpico, and I. D. Mayergoyz. Nonlinear magnetization dynamics under circularly polarized field. Physical Review Letters, 86(4):724, 2001.

[11] J. L. Blue and M. Scheinfein. Using multipoles decreases computation time for magnetostatic self-energy. IEEE Transactions on Magnetics, 27(6):4778-4780, 1991.

[12] F. Brezzi, K. Lipnikov, and V. Simoncini. A family of mimetic finite difference methods on polygonal and polyhedral meshes. Mathematical Models and Methods in Applied Sciences, 15(10):1533-1551, 2005.

[13] X. Brunotte, G. Meunier, and J.-F. Imhoff. Finite element modeling of unbounded problems using transformations: a rigorous, powerful and easy solution. IEEE Transactions on Magnetics, 28(2):1663-1666, 1992. 
[14] I. Cimrák. Error estimates for a semi-implicit numerical scheme solving the LandauLifshitz equation with an exchange field. IMA Journal of Numerical Analysis, 25(3):611$634,2005$.

[15] I. Cimrák. A survey on the numerics and computations for the Landau-Lifshitz equation of micromagnetism. Archives of Computational Methods in Engineering, 15(3):1-37, 2007.

[16] I. Cimrák. Convergence result for the constraint preserving mid-point scheme for micromagnetism. Journal of Computational and Applied Mathematics, 228(1):238-246, 2009.

[17] M. d'Aquino, C. Serpico, and G. Miano. Geometrical integration of Landau-LifshitzGilbert equation based on the mid-point rule. Journal of Computational Physics, 209(2):730-753, 2005.

[18] L. Exl, W. Auzinger, S. Bance, M. Gusenbauer, F. Reichel, and T. Schrefl. Fast stray field computation on tensor grids. Journal of Computational Physics, 231(7):2840-2850, 2012.

[19] R. D. Falgout and U. M. Yang. hypre: A library of high performance preconditioners. In International Conference on Computational Science, pages 632-641. Springer, 2002.

[20] J. Fidler and T. Schrefl. Micromagnetic modelling-the current state of the art. Journal of Physics D: Applied Physics, 33(15):R135, 2000.

[21] D. Fredkin and T. Koehler. Hybrid method for computing demagnetizing fields. IEEE Transactions on Magnetics, 26(2):415-417, 1990.

[22] A. Fuwa, T. Ishiwata, and M. Tsutsumi. Finite difference scheme for the LandauLifshitz equation. Japan Journal of Industrial and Applied Mathematics, 29(1):83-110, 2012.

[23] C. J. García-Cervera. Numerical micromagnetics: A review. Bol. Soc. Esp. Mat. Apl., 39:103-135, 2007.

[24] C. J. García-Cervera and W. E. Improved gauss-seidel projection method for micromagnetics simulations. IEEE transactions on magnetics, 39(3):1766-1770, 2003.

[25] C. J. García-Cervera, Z. Gimbutas, and W. E. Accurate numerical methods for micromagnetics simulations with general geometries. Journal of Computational Physics, 184(1):37-52, 2003.

[26] C. J. García-Cervera and A. M. Roma. Adaptive mesh refinement for micromagnetics simulations. IEEE Transactions on Magnetics, 42(6):1648-1654, 2006.

[27] S. Gustafson, K. Nakanishi, and T.-P. Tsai. Asymptotic Stability, Concentration, and Oscillation in Harmonic Map Heat-Flow, Landau-Lifshitz, and Schrödinger Maps on $\mathbb{R}^{2}$. Communications in Mathematical Physics, 300(1):205-242, 2010. 
[28] N. Hayashi, K. Saito, and Y. Nakatani. Calculation of demagnetizing field distribution based on fast fourier transform of convolution. Japanese Journal of Applied Physics, 35:6065-6073., 1996.

[29] J. Hyman and M. Shashkov. The approximation of boundary conditions for mimetic finite difference methods. Computers and Mathematics with Applications, 36:79-99, 1998.

[30] J. S. Jiang, H. G. Kaper, and G. K. Leaf. Hysteresis in layered spring magnets. Discrete and Continuous Dynamical Systems Series B, 1:219-323, 2001.

[31] P. S. Krishnaprasad and X. Tan. Cayley transforms in micromagnetics. Physica B: Condensed Matter, 306(1):195-199, 2001.

[32] E. Kritsikis, A. Vaysset, L. Buda-Prejbeanu, F. Alouges, and J.-C. Toussaint. Beyond first-order finite element schemes in micromagnetics. Journal of Computational Physics, 256:357-366, 2014.

[33] M. Kruzík and A. Prohl. Recent developments in the modeling, analysis, and numerics of ferromagnetism. SIAM Review, 48(3):439-483, 2006.

[34] D. Lewis and N. Nigam. Geometric integration on spheres and some interesting applications. Journal of Computational and Applied Mathematics, 151(1):141-170, 2003.

[35] S.-Z. Lin, C. Reichhardt, and A. Saxena. Manipulation of skyrmions in nanodisks with a current pulse and skyrmion rectifier. Applied Physics Letters, 102(22):222405, 2013.

[36] K. Lipnikov. Monotonicity conditions in the mimetic finite diffence method. In J.Fort, J.Fürst, J.Halama, R.Herbin, and F.Hubert, editors, Finite Volumes for Complex Applications VI Problems \& Perspectives, volume 1 of Springer Proceedings in Mathematics, pages 653-662. Springer, 2011.

[37] K. Lipnikov, G. Manzini, and M. Shashkov. Mimetic finite difference method. J. Comp. Phys., 257:1163-1227, 2014.

[38] K. Lipnikov, G. Manzini, and D. Svyatskiy. Analysis of the monotonicity conditions in the mimetic finite difference method for elliptic problems. Journal of Computational Physics, 230(7):2620-2642, 2011.

[39] B. Livshitz, A. Boag, H. N. Bertram, and V. Lomakin. Nonuniform grid algorithm for fast calculation of magnetostatic interactions in micromagnetics. Journal of Applied Physics, 105(7):07D541, 2009.

[40] H. Long, E. Ong, Z. Liu, and E. Li. Fast Fourier transform on multipoles for rapid calculation of magnetostatic fields. IEEE Transactions on Magnetics, 42(2):295-300, 2006 .

[41] J. E. Miltat and M. J. Donahue. Numerical Micromagnetics: Finite difference methods. Handbook of magnetism and advanced magnetic materials, 2007. 
[42] N. Popović and D. Praetorius. Applications of $\mathcal{H}$-matrix techniques in micromagnetics. Computing, 74(3):177-204, 2005.

[43] A. Prohl. Computational Micromagnetism. Advances in Numerical Mathematics. Teubner, Stuttgar, 2001.

[44] T. Schrefl. Finite elements in numerical micromagnetics: part i: granular hard magnets. Journal of Magnetism and Magnetic Materials, 207(1):45-65, 1999.

[45] I. Tsukerman, A. Plaks, and H. N. Bertram. Multigrid methods for computation of magnetostatic fields in magnetic recording problems. Journal of Applied Physics, 83(11):6344-6346, 1998.

[46] X.-P. Wang. Numerical methods for the Landau-Lifshitz equation. SIAM Journal on Numerical Analysis, 38(5):1647-1665, 2000.

[47] X.-P. Wang, C. J. García-Cervera, and W. E. A Gauss-Seidel projection method for micromagnetics simulations. Journal of Computational Physics, 171(1):357-372, 2001.

[48] X.-P. Wang, K. Wang, and W. E. Simulations of 3-d domain wall structures in thin films. Discrete and Continuous Dynamical Systems Series B, 6(2):373, 2006.

[49] S. W. Yuan and H. N. Bertram. Fast adaptive algorithms for micromagnetics. IEEE Transactions on Magnetics, 28(5):2031-2036, 1992. 